\title{
An integral formula for Riemannian $G$-structures with applications to almost Hermitian and almost contact structures
}

\author{
Kamil Niedziałomski ${ }^{1}$
}

Received: 14 October 2018 / Accepted: 9 May 2019 / Published online: 20 May 2019

(c) The Author(s) 2019

\begin{abstract}
For a Riemannian $G$-structure, we compute the divergence of the vector field induced by the intrinsic torsion. Applying the Stokes theorem, we obtain the integral formula on a closed oriented Riemannian manifold, which we interpret in certain cases. We focus on almost Hermitian and almost contact metric structures.
\end{abstract}

Keywords Integral formula Intrinsic torsion - Almost Hermitian structures · Almost contact metric structures

Mathematics Subject Classification 53C10 $\cdot 53 \mathrm{C} 24 \cdot 53 \mathrm{C} 43$

\section{Introduction}

Equipping an $n$-dimensional manifold $M$ with a Riemannian metric $g$ is equivalent to the reduction of a frame bundle $L(M)$ to the orthogonal frame bundle $O(M)$, i.e., to action of a structure group $O(n)$. Assuming moreover that $M$ is oriented, we can consider the bundle $S O(M)$ of oriented orthonormal frames. Existence of additional geometric structure can be considered as a reduction of a structure group $S O(n)$ to a certain subgroup $G$. For example, almost Hermitian structure gives $U\left(\frac{n}{2}\right)$-structure, almost contact metric structure is just a $U\left(\frac{n-1}{2}\right) \times 1$-structure, etc.

If $\nabla$ is the Levi-Civita connection of $(M, g)$, we may measure the defect of $\nabla$ to be a $G$-connection. This leads to the notion of an intrinsic torsion. If this (1,2)-tensor vanishes (in such case, we say that a $G$-structure is integrable), then $\nabla$ is a $G$-connection, which implies that the holonomy group is contained in $G$. We may classify non-integrable geometries by finding the decomposition of the space of all possible intrinsic torsions into irreducible $G$ modules. This approach was initiated by Gray and Hervella for $U\left(\frac{n}{2}\right)$-structures [14] and

Kamil Niedziałomski

kamil.niedzialomski@wmii.uni.lodz.pl

1 Department of Mathematics and Computer Science, University of Łódź, ul. Banacha 22, 90-238 Lodz, Poland 
later considered for other structures by many authors [7,8,11,20,21]. Each, so-called, GrayHervella class, gives some restrictions on the curvature.

One possible approach to curvature restrictions on compact $G$-structures can be achieved by obtaining integral formulas relating considered objects. This has been firstly done, in a general case, by Bor and Hernández Lamoneda [5]. They use Bochner-type formula for forms being stabilizers of each considered subgroup in $S O(n)$. They obtained integral formulas for $G=U\left(\frac{n}{2}\right), S U\left(\frac{n}{2}\right), G_{2}$ and $\operatorname{Spin}_{7}$ and continued this approach for $\operatorname{Sp}(n) \operatorname{Sp}(1)$ in [6]. The case $G=U\left(\frac{n-1}{2}\right) \times 1$ has been studied later in [12] by other authors.

In this article, we show how mentioned formulas can be obtained in a different way. The nice feature of our approach is that the main integral formula

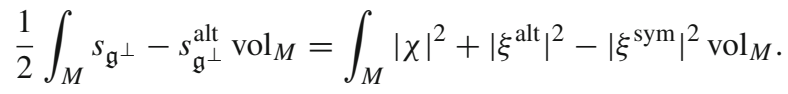

is valid for any $G$-structure on closed $M$ for compact $G \subset S O(n)$. Let us roughly describe the approach and all used objects in this formula. We consider, so-called, characteristic vector field $\chi=\sum_{i} \xi_{e_{i}} e_{i}$ induced by the intrinsic torsion $\xi$ and calculate its divergence. $\xi^{\text {alt }}$ and $\xi^{\text {sym }}$ denote the skew-symmetric and symmetric components of $\xi, \xi_{X}^{\text {alt }} Y=\frac{1}{2}\left(\xi_{X} Y-\xi_{Y} X\right)$, $\xi_{X}^{\text {sym }} Y=\frac{1}{2}\left(\xi_{X} Y+\xi_{Y} X\right)$, whereas $s_{\mathfrak{g}^{\perp}}$ and $s_{\mathfrak{g}^{\perp}}^{\text {alt }}$ are, in a sense, $\mathfrak{g}^{\perp}$ components of a scalar curvature (see the following sections for more details). For some Gray-Hervella classes, the characteristic vector field vanishes, and then we get point-wise formula relating an intrinsic torsion to a curvature.

We concentrate on almost Hermitian and almost contact metric structures. In the way described above, we recover many well-known relations. Let us state some of the consequences of the main integral formula (the objects used in these statements will be defined in appropriate sections):

1. Assume $(M, g, J)$ is closed Hermitian manifold of Gray-Hervella type $\mathcal{W}_{4}$ such that $s=s^{*}$, where $s$ is a scalar curvature and $s^{*}$ is a $*$-scalar curvature. Then, $M$ is Kähler (compare [22]).

2. On a closed $S U(n)$-structure of type $\mathcal{W}_{1} \oplus \mathcal{W}_{5}$, we have $\int_{M} s=5 \int_{M} s^{*}$.

3. Let $(M, g, \varphi, \eta, \zeta)$ be an almost contact metric structure with the intrinsic torsion $\xi \in \mathcal{D}_{2}$. Then,

$$
\operatorname{div}\left(\nabla_{\zeta} \zeta\right)=\frac{1}{2} s_{\mathfrak{u}(n)^{\perp}}^{\text {alt }}+\frac{1}{2} \operatorname{Ric}(\zeta, \zeta)-\frac{1}{4}\left(s-s^{*}\right),
$$

where $s$ is a scalar curvature and $s^{*}$ is an associated $*$-scalar curvature.

In the end, we consider some examples focusing on (reductive) homogeneous spaces. We show, which is an immediate consequence of the formula for the Levi-Civita connection, that in these examples the characteristic vector field vanishes. Hence, the main divergence formula is point-wise.

\section{Intrinsic torsion}

Let $(M, g)$ be an oriented Riemannian manifold. Denote by $S O(M)$ the bundle of oriented frames over $M$. Let $\nabla$ be the Levi-Civita connection of $g$, and let $\omega$ be the induced connection form. Let $G \subset S O(n)$, where $n=\operatorname{dim} M$, be a closed subgroup. Then, on the level of Lie algebras, we have the following decomposition:

$$
\mathfrak{s o}(n)=\mathfrak{g} \oplus \mathfrak{g}^{\perp}, \quad \operatorname{ad}(G) \mathfrak{g}^{\perp} \subset \mathfrak{g}^{\perp},
$$


where the orthogonal complement is taken with respect to the Killing form. Hence, $\omega$ decomposes as

$$
\omega=\omega_{\mathfrak{g}} \oplus \omega_{\mathfrak{g}^{\perp}}
$$

where $\omega_{\mathfrak{g}}$ is a connection form in the $G$-reduction $P \subset S O(M)$, if such exists, and therefore defines a Riemannian connection $\nabla^{G}$ on $M$. The difference

$$
\xi_{X} Y=\nabla_{X}^{G} Y-\nabla_{X} Y, \quad X, Y \in T M,
$$

defines a $(1,2)$-tensor called the intrinsic torsion of a $G$-structure. $\xi$ satisfies some skewsymmetry conditions by the fact that $\xi_{X} \in \mathfrak{g}^{\perp}(T M) \subset \mathfrak{s o}(T M)$ where $\mathfrak{g}^{\perp}(T M)$ is the associated bundle of the form $P \times_{\mathrm{ad}(\mathrm{G})} \mathfrak{g}^{\perp}$. In particular,

$$
g\left(\xi_{X} Y, Z\right)=-g\left(Y, \xi_{X} Z\right), \quad X, Y, Z \in T M .
$$

By a definition, the intrinsic torsion measures the defect of the Levi-Civita connection to be a $G$-connection. In particular, if $\xi$ vanishes, then the holonomy of $\nabla$ is contained in $G$. The study of the intrinsic torsion and its decomposition into irreducible summands was initiated by Gray and Hervella in the case of $G=U\left(\frac{n}{2}\right)$ [14]. Since then, other possible cases, mainly coming from the Berger classification of non-symmetric irreducible holonomy groups, have been considered (see, for example, $[7,8,11,20,21]$ ).

\section{An integral formula}

Let $(M, g)$ be an oriented Riemannian manifold with the Levi-Civita connection $\nabla$. Assume $M$ is a $G$-structure, with $G \subset S O(n)$, and let $\xi$ be the associated intrinsic torsion. Define a vector field $\chi=\chi^{G}$ by

$$
\chi=\sum_{i} \xi_{e_{i}} e_{i}
$$

where $\left(e_{i}\right)$ is any orthonormal basis. We call $\chi$ the characteristic vector field of a $G$-structure $M$. Notice that if $\xi$ is skew-symmetric with respect to $X$ and $Y$, then $\chi$ vanishes. This is the case, for example, for nearly Kähler manifolds (see the following sections). Additionally,

$$
g(\chi, X)=-\sum_{i} g\left(e_{i}, \xi_{e_{i}} X\right)=\operatorname{div} X-\operatorname{div}^{G} X .
$$

Thus, vanishing of the characteristic vector field is equivalent to the fact that divergences with respect to $\nabla$ and $\nabla^{G}$ coincide. Moreover, put

$$
\xi_{X}^{\text {alt }} Y=\frac{1}{2}\left(\xi_{X} Y-\xi_{Y} X\right) \text { and } \xi_{X}^{\text {sym }} Y=\frac{1}{2}\left(\xi_{X} Y+\xi_{Y} X\right) .
$$

In this section, we compute the divergence of $\chi$ with respect to $\nabla$. First, let us recall well-known curvature identities involving the intrinsic torsion [12]:

$$
\begin{aligned}
R(X, Y)_{\mathfrak{g}} & =R^{G}(X, Y)+\left[\xi_{X}, \xi_{Y}\right]_{\mathfrak{g}}, \\
R(X, Y)_{\mathfrak{g}^{\perp}} & =-\left(\nabla_{X} \xi\right)_{Y}+\left(\nabla_{Y} \xi\right)_{X}-2\left[\xi_{X}, \xi_{Y}\right]+\left[\xi_{X}, \xi_{Y}\right]_{\mathfrak{g}^{\perp}},
\end{aligned}
$$

where $R$ and $R^{G}$ are the curvature tensors of $\nabla$ and $\nabla^{G}$, respectively. We use the following convention for the curvature $R(X, Y)=\left[\nabla_{X}, \nabla_{Y}\right]-\nabla_{[X, Y]}$. Thus,

$$
R^{G}(X, Y)=R(X, Y)+\left(\nabla_{X} \xi\right)_{Y}-\left(\nabla_{Y} \xi\right)_{X}+\left[\xi_{X}, \xi_{Y}\right] .
$$


Denote by $s$ and $s^{G}$ the scalar curvatures of $R$ and $R^{G}$, respectively.

Proposition 1 On an oriented $G$-structure $M$, we have

$$
2 \operatorname{div} \chi=s^{G}-s+|\chi|^{2}+\left|\xi^{\text {alt }}\right|^{2}-\left|\xi^{\text {sym }}\right|^{2} .
$$

Proof By (5), we have

$$
s^{G}=s+\sum_{i, j} g\left(\left(\nabla_{e_{i}} \xi\right)_{e_{j}} e_{j}, e_{i}\right)-\sum_{i, j} g\left(\left(\nabla_{e_{j}} \xi\right)_{e_{i}} e_{j}, e_{i}\right)+\sum_{i, j} g\left(\left[\xi_{e_{i}}, \xi_{e_{j}}\right] e_{j}, e_{i}\right) .
$$

Notice that $\left(\nabla_{X} \xi\right)_{Y}$ is skew-symmetric, since $\xi_{X}$ is skew-symmetric,

$$
\begin{aligned}
g\left(\left(\nabla_{X} \xi\right)_{Y} Z, W\right) & =g\left(\nabla_{X} \xi_{Y} Z, W\right)-g\left(\xi_{\nabla_{X} Y} Z, W\right)-g\left(\xi_{Y} \nabla_{X} Z, W\right) \\
& =-X\left(Z, \xi_{Y} W\right)-g\left(\xi_{Y} Z, \nabla_{X} W\right)+g\left(Z, \xi_{\nabla_{X} Y} W\right)+\left(\nabla_{X} Z, \xi_{Y} W\right) \\
& =-g\left(Z, \nabla_{X} \xi_{Y} W\right)+g\left(Z, \xi_{Y} \nabla_{X} W\right)+g\left(Z, \xi_{\nabla_{X} Y} W\right) \\
& =-g\left(Z,\left(\nabla_{X} \xi\right)_{Y} W\right) .
\end{aligned}
$$

Thus, the first sum and second sum on the right-hand side of (7) are opposite. Moreover,

$$
\operatorname{div} \chi=\sum_{i, j} g\left(\nabla_{e_{i}} \xi_{e_{j}} e_{j}, e_{i}\right)=\sum_{i, j} g\left(\left(\nabla_{e_{i}} \xi\right)_{e_{j}} e_{j}, e_{i}\right),
$$

since

$$
\begin{aligned}
& \sum_{i, j}\left(g\left(\xi_{\nabla_{e_{i}} e_{j}} e_{j}, e_{i}\right)+g\left(\xi_{e_{j}} \nabla_{e_{i}} e_{j}, e_{i}\right)\right) \\
& =\sum_{i, j, k} g\left(\nabla_{e_{i}} e_{j}, e_{k}\right) g\left(\xi_{e_{k}} e_{j}, e_{i}\right)+\sum_{i, j} g\left(\xi_{e_{j}} \nabla_{e_{i}} e_{j}, e_{i}\right) \\
& =-\sum_{i, j, k} g\left(e_{j}, \nabla_{e_{i}} e_{k}\right) g\left(\xi_{e_{k}} e_{j}, e_{i}\right)+\sum_{i, j} g\left(\xi_{e_{j}} \nabla_{e_{i}} e_{j}, e_{i}\right) \\
& =0 .
\end{aligned}
$$

Let us compute the last term in (7),

$$
\begin{aligned}
\sum_{i, j} g\left(\left[\xi_{e_{i}}, \xi_{e_{j}}\right] e_{j}, e_{i}\right) & =\sum_{i, j} g\left(\xi_{e_{i}} \xi_{e_{j}} e_{j}, e_{i}\right)-g\left(\xi_{e_{j}} \xi_{e_{i}} e_{j}, e_{i}\right) \\
& =-|\chi|^{2}+\sum_{i, j} g\left(\xi_{e_{j}} e_{i}, \xi_{e_{i}} e_{j}\right) \\
& =-|\chi|^{2}+\left|\xi^{\mathrm{sym}}\right|^{2}-\left|\xi^{\text {alt }}\right|^{2} .
\end{aligned}
$$

Substituting (8) and (9) into (7), we get (6).

We will improve the above divergence formula a little bit, by getting rid of the component $s^{G}$ and replacing it by $\mathfrak{g}^{\perp}$-component of $s$ and some additional term, which vanishes in some cases. Namely, denote by $s_{\mathfrak{g}^{\perp}}^{\text {alt }}$ the following quantity:

$$
s_{\mathfrak{g}^{\perp}}^{\mathrm{alt}}=\sum_{i, j} g\left(\left[\xi_{e_{i}}, \xi_{e_{j}}\right]_{\mathfrak{g}^{\perp}} e_{j}, e_{i}\right) .
$$


Proposition 2 On an oriented $G$-structure $M$, we have

$$
\operatorname{div} \chi=\frac{1}{2} s_{\mathfrak{g}^{\perp}}^{\text {alt }}-\frac{1}{2} s_{\mathfrak{g}^{\perp}}+|\chi|^{2}+\left|\xi^{\text {alt }}\right|^{2}-\left|\xi^{\mathrm{sym}}\right|^{2} .
$$

If $M$ is, additionally, closed, then the following integral formula holds

$$
\frac{1}{2} \int_{M} s_{\mathfrak{g}^{\perp}}-s_{\mathfrak{g}^{\perp}}^{\text {alt }} \text { vol }=\int_{M}|\chi|^{2}+\left|\xi^{\text {alt }}\right|^{2}-\left|\xi^{\text {sym }}\right|^{2} \text { vol. }
$$

Proof By (4) and (9), we have

$$
\begin{aligned}
s_{\mathfrak{g}} & =s^{G}+\sum_{i, j} g\left(\left[\xi_{e_{i}}, \xi_{e_{j}}\right] e_{j}, e_{i}\right)-\sum_{i, j} g\left(\left[\xi_{e_{i}}, \xi_{e_{j}}\right]_{\mathfrak{g}^{\perp}} e_{j}, e_{i}\right) \\
& =s^{G}-|\chi|^{2}-\left|\xi^{\text {alt }}\right|^{2}+\left|\xi^{\mathrm{sym}}\right|^{2}-\sum_{i, j} g\left(\left[\xi_{e_{i}}, \xi_{e_{j}}\right]_{\mathfrak{g}^{\perp}} e_{j}, e_{i}\right) .
\end{aligned}
$$

Since $s=s_{\mathfrak{g}}+s_{\mathfrak{g}^{\perp}},(6)$ can be rewritten in form (10).

Remark 1 Notice that elements

$$
|\chi|^{2}, \quad\left|\xi^{\text {alt }}\right|^{2}, \quad\left|\xi^{\mathrm{sym}}\right|^{2}
$$

are quadratic invariants of the representation of $S O(n)$ in the space of $(1,2)$-tensors with the symmetries of the intrinsic torsion, i.e., the space $T^{*} M \otimes \mathfrak{s o}(T M)$ [14]. This implies that $|\xi|^{2}$ and $\left|\xi^{\text {alt }}\right|^{2}-\left|\xi^{\text {sym }}\right|^{2}$ are also quadratic invariants. Thus, for an irreducible submodule $\mathcal{U}$ of the representation $T^{*} M \otimes \mathfrak{s o}(T M)$, since the space of its quadratic invariants is one-dimensional [4], then the number

$$
E_{\mathcal{U}}=\left|\chi^{\mathcal{U}}\right|^{2}+\left|\xi^{\mathcal{U}, \text { alt }}\right|^{2}-\left|\xi^{\mathcal{U}, \text { sym }}\right|^{2}
$$

is a constant multiple of $\left|\xi^{\mathcal{U}}\right|^{2}$. Here, $\xi^{\mathcal{U}}$ denotes the $\mathcal{U}$-component of $\xi$ with respect to decomposition into irreducible summands. This approach is also valid for any irreducible module $G$-module in the space of possible intrinsic torsions. This kind of approach was used in [5] to get integral formulas for many $G$-structures.

We have an immediate consequence of the formula (10).

Corollary 1 Assume $M$ is an oriented $G$-structure, where $G=U\left(\frac{n}{2}\right), n$ even, or $G=$ $S O(m) \times S O(n-m)$. If the characteristic vector field vanishes, then

$$
\frac{1}{2} s_{\mathfrak{g}^{\perp}}=\left|\xi^{\mathrm{alt}}\right|^{2}-\left|\xi^{\mathrm{sym}}\right|^{2} .
$$

In particular, if the intrinsic torsion is totally skew-symmetric, then

$$
s_{\mathfrak{g}^{\perp}}=2|\xi|^{2} \geq 0
$$

with the equality if and only if the $G$-structure $M$ is integrable (i.e., $\xi=0$ ).

Proof For the listed choices of $G$, we have $\left[\mathfrak{g}^{\perp}, \mathfrak{g}^{\perp}\right] \subset \mathfrak{g}$; thus, $s_{\mathfrak{g}^{\perp}}^{\text {alt }}$ vanishes.

The consequences of the integral formula will be presented in the following section for certain choices of $G$. 


\section{Applications to certain Riemannian G-structures}

In this section, we rewrite formulas (10) and (11) for certain $G$-structures. We also give some applications of these relations. We will show that obtained formulas are consistent with the Bochner-type formulas obtained, using representation theory, in [5].

\subsection{Almost product structures}

We show that the divergence and integral formulas obtained in the previous section agree with the Walczak formulas [23]. Since this integral formula has found many applications, we will only concentrate on deriving it from (11) and state its one corollary, which will be needed later.

Let $(M, g)$ be an oriented Riemannian manifold, with two complementary orthogonal oriented distributions $\mathcal{D}$ and $\mathcal{D}^{\perp}$, i.e., $T M=\mathcal{D} \oplus \mathcal{D}^{\perp}$. Thus, the bundle of oriented orthonormal frames $S O(M)$ has a reduction to a subgroup $S O(m) \times S O(n-m) \subset S O(n)$, where $m=\operatorname{dim} \mathcal{D}$. On the level of Lie algebras

$$
\mathfrak{s o}(n)=(\mathfrak{s o}(m) \oplus \mathfrak{s o}(n-m)) \oplus \mathfrak{m},
$$

where

$$
\mathfrak{m}=(\mathfrak{s o}(m) \oplus \mathfrak{s o}(n-m))^{\perp}=\left\{\left(\begin{array}{cc}
0 & A \\
-A^{\top} & 0
\end{array}\right)\right\}
$$

and $A$ is $m \times(n-m)$ matrix. Let $\nabla$ be the Levi-Civita connection of $g$. Since the orthogonal projection to $\mathfrak{m}$ is just a restriction to non-diagonal blocks, it follows that the intrinsic torsion equals

$$
\xi_{X} Y=-\left(\nabla_{X} Y^{\top}\right)^{\perp}-\left(\nabla_{X} Y^{\perp}\right)^{\top},
$$

where $Y^{\top}$ and $Y^{\perp}$ denote the components of $Y$ in $\mathcal{D}$ and $\mathcal{D}^{\perp}$. Notice that $\xi$ is made of shape operators and fundamental forms of distributions $\mathcal{D}$ and $\mathcal{D}^{\perp}$. Recall that the second fundamental form, for example, of $\mathcal{D}$ is a $(1,2)$-symmetric tensor $B=B^{\mathcal{D}}$ of the form

$$
B(X, Y)=\frac{1}{2}\left(\nabla_{X} Y+\nabla_{Y} X\right)^{\perp}, \quad X, Y \in \mathcal{D} .
$$

Additionally, we will use integrability tensor $T=T^{\mathcal{D}}$ being just

$$
T(X, Y)=\frac{1}{2}[X, Y]^{\perp}, \quad X, Y \in \mathcal{D} .
$$

Notice that $B(X, Y)+T(X, Y)=\left(\nabla_{X} Y\right)^{\perp}$; hence, $B$ and $T$ are symmetrization and alternation of (a minus of) a part of the intrinsic torsion reduced to $\mathcal{D}$.

For an orthonormal basis $\left(e_{i}\right)$ adapted to the decomposition $\mathcal{D} \oplus \mathcal{D}^{\perp}$, denote by $e_{A}$ elements of $\left(e_{i}\right)$ in $\mathcal{D}$ and by $e_{\alpha}$ elements of $\left(e_{i}\right)$ in $\mathcal{D}^{\perp}$. The characteristic vector field $\chi$ equals

$$
\chi=-\sum_{A}\left(\nabla_{e_{A}} e_{A}\right)^{\perp}-\sum_{\alpha}\left(e_{\alpha} e_{\alpha}\right)^{\top}=-H-H^{\perp},
$$

where $H$ and $H^{\perp}$ are mean curvature vectors of $\mathcal{D}$ and $\mathcal{D}^{\perp}$, respectively.

We may now state and show that the Walczak formula [23] is an integral formula (11) for $G=S O(m) \times S O(n-m)$. 
Proposition 3 ([23]) On a closed Riemannian manifold equipped with a pair of complementary orthogonal and oriented distributions, the following Walczak integral formula holds

$$
\int_{M} s_{\text {mix }}=\int_{M}|H|^{2}+\left|H^{\perp}\right|^{2}+|T|^{2}+\left|T^{\perp}\right|^{2}-|B|^{2}-\left|B^{\perp}\right|^{2},
$$

where $s_{\operatorname{mix}}$ is a mixed scalar curvature defined by

$$
s_{\text {mix }}=\sum_{A, \alpha} g\left(R\left(e_{A}, e_{\alpha}\right) e_{\alpha}, e_{A}\right)
$$

Proof Since

$$
\sum_{A, \alpha}\left|\left(\nabla_{e_{\alpha}} e_{A}\right)^{\perp}\right|^{2}=\sum_{A, \alpha, \beta} g\left(\nabla_{e_{\alpha}} e_{A}, e_{\beta}\right)^{2}=\sum_{A, \alpha, \beta} g\left(\nabla_{e_{\alpha}} e_{\beta}, e_{A}\right)^{2}=\sum_{\alpha, \beta}\left|\left(\nabla_{e_{\alpha}} e_{\beta}\right)^{\top}\right|^{2}
$$

and analogously interchanging $e_{\alpha}$ with $e_{A}$, then

$$
\left|\xi^{\text {alt }}\right|^{2}=|T|^{2}+\left|T^{\perp}\right|^{2}+\frac{1}{4} \sum_{A, B}\left|\left(\nabla_{e_{A}} e_{B}\right)^{\perp}\right|^{2}+\frac{1}{4} \sum_{\alpha, \beta}\left|\left(\nabla_{e_{\alpha}} e_{\beta}\right)^{\top}\right|^{2}
$$

and

$$
\left|\xi^{\mathrm{sym}}\right|^{2}=|B|^{2}+\left|B^{\perp}\right|^{2}+\frac{1}{4} \sum_{\alpha, \beta}\left|\left(\nabla_{e_{\alpha}} e_{\beta}\right)^{\top}\right|^{2}
$$

Moreover,

$$
s_{\mathfrak{m}}=\sum_{i, j} g\left(R\left(e_{i}, e_{j}\right)_{\mathfrak{m}} e_{j}, e_{i}\right)=2 \sum_{A, \alpha} g\left(R\left(e_{A}, e_{\alpha}\right) e_{\alpha}, e_{A}\right)=2 s_{\text {mix }} .
$$

Putting all these facts together (10) implies Walczak divergence formula [23]

$$
-\operatorname{div}\left(H+H^{\perp}\right)=-s_{\text {mix }}+|H|^{2}+\left|H^{\perp}\right|^{2}+|T|^{2}+\left|T^{\perp}\right|^{2}-|B|^{2}-\left|B^{\perp}\right|^{2} .
$$

Assuming $M$ is closed, the Walczak integral formula holds.

Formula (13) has found many applications. Let us only state one of its consequences for $\mathcal{D}$ of codimension 1 , since it will be used in one of forthcoming subsections. In this case, clearly, $T^{\perp}=0$ and $B^{\perp}=H^{\perp}$. Denoting the unit positively oriented vector field orthogonal to $\mathcal{D}$ by $\zeta$, we have $\chi=(\operatorname{div} \zeta) \zeta-\nabla_{\zeta} \zeta$. Moreover,

$$
s_{\text {mix }}=\sum_{A} g\left(R\left(e_{A}, \zeta\right) \zeta, e_{A}\right)=\operatorname{Ric}(\zeta, \zeta) .
$$

Therefore, (14) and (13) can be rewritten in the following well-known way.

Proposition 4 On a Riemannian manifold with an orientable codimension one distribution $\mathcal{D}$, we have the following divergence formula:

$$
\operatorname{div}\left(-(\operatorname{div} \zeta) \zeta+\nabla_{\zeta} \zeta\right)=\operatorname{Ric}(\zeta, \zeta)-(\operatorname{div} \zeta)^{2}-|T|^{2}+|B|^{2}
$$

and, assuming $M$ is closed, the following integral formula

$$
\int \operatorname{Ric}(\zeta, \zeta) \operatorname{vol}_{M}=\int_{M}(\operatorname{div} \zeta)^{2}+|T|^{2}-|B|^{2} \operatorname{vol}_{M}
$$




\subsection{Almost Hermitian structures}

Assume $(M, g, J)$ is an oriented Riemannian manifold with an almost complex structure $J$, i.e., $J^{2}=-\mathrm{id}_{T M}$, which is Hermitian, i.e., $g(J X, J Y)=g(X, Y)$ for $X, Y \in T M$. Then, $(M, g, J)$ is of even dimension $2 n$ and induces an $U(n)$-structure. On the level of Lie algebras, we have

$$
\mathfrak{s o}(2 n)=\mathfrak{u}(n) \oplus \mathfrak{u}(n)^{\perp},
$$

where

$$
\mathfrak{u}(n)=\{A \in \mathfrak{s o}(n) \mid A J=J A\}, \quad \mathfrak{u}(n)^{\perp}=\{A \in \mathfrak{s o}(n) \mid A J=-J A\} .
$$

In particular, $\left[\mathfrak{u}(n)^{\perp}, \mathfrak{u}(n)^{\perp}\right] \subset \mathfrak{u}(n)$, thus $s_{\mathfrak{u}(n)^{\perp}}^{\text {alt }}=0$. The orthogonal projection from $\mathfrak{s o}(n)$ to $\mathfrak{u}(n)^{\perp}$ equals $A \mapsto \frac{1}{2}(A+J A J)$. Thus, the $\mathfrak{u}(n)$-component of $R$ is given by

$$
R(X, Y)_{\mathfrak{u}(n)}=\frac{1}{2}(R(X, Y)+J \circ R(X, Y) \circ J) .
$$

Moreover, the intrinsic torsion, being informally the projection of $-\nabla$ to $\mathfrak{u}(n)^{\perp}$, is given by the formula

$$
\xi_{X} Y=-\frac{1}{2} J\left(\nabla_{X} J\right) Y
$$

Hence, the characteristic vector field $\chi$ is the following

$$
\chi=-\frac{1}{2} J(\operatorname{div} J) .
$$

Let us describe the intrinsic torsion with the use of the Nijenhuis tensor $N$ and the Kähler form $\Omega$. Recall that

$$
\begin{aligned}
N(X, Y) & =[J X, J Y]-J[X, J Y]-J[J X, Y]-[X, Y] \\
& =\left(\nabla_{X} J\right) J Y-\left(\nabla_{Y} J\right) J X+\left(\nabla_{J X} J\right) Y-\left(\nabla_{J Y} J\right) X
\end{aligned}
$$

and

$$
\Omega(X, Y)=g(X, J Y) .
$$

It is a famous theorem by Newlander and Nirenberg that vanishing of the Nijenhuis tensor is equivalent to integrability of $J$, i.e., existence of complex coordinates adapted to $J$. In can be shown [2] that

$$
4 g\left(\xi_{X} Y, Z\right)=d \Omega(X, Y, J Z)+d \Omega(X, J Y, Z)-g(N(Y, Z), X) .
$$

Unfortunately, this shows that $\xi$ has no particular symmetries and using (19), it is hard to give nice interpretations for the symmetrized and skew-symmetrized intrinsic torsion $\xi^{\mathrm{sym}}$ and $\xi^{\text {alt }}$, respectively. Therefore, it is convenient to consider some restrictions or decomposition of the intrinsic torsion. The space of all possible intrinsic torsions is, in this case, $T^{*} M \otimes$ $\mathfrak{u}(n)^{\perp}(T M)$. Decomposing this space into irreducible modules with respect to $U(n)$-action, we get so-called Gray-Hervella classes [14]

$$
T^{*} M \otimes \mathfrak{u}(n)^{\perp}(T M)=\mathcal{W}_{1} \oplus \mathcal{W}_{2} \oplus \mathcal{W}_{3} \oplus \mathcal{W}_{4},
$$


where each class can be characterized as follows:

$\mathcal{W}_{1}: \xi_{X} Y=-\xi_{Y} X$, in particular, $\chi=0$.

$\mathcal{W}_{2}: g\left(\xi_{X} Y, Z\right)+g\left(\xi_{Z} X, Y\right)+g\left(\xi_{Y} Z, X\right)=0$. Then $\chi=0$.

$\mathcal{W}_{3}: \xi_{X} Y=\xi_{J X}(J Y)$ and $\chi=0$,

$\mathcal{W}_{4}:-4 \xi_{X} Y=\theta(Y) X+\theta(J Y) J X-g(X, Y) \theta^{\sharp}-g(X, J Y) J \theta^{\sharp}, \theta \in \Gamma\left(T^{*} M\right)$.

In the characterization of $\mathcal{W}_{4}, \theta$ is a one-form often called the Lee form.

The following proposition contains well-known and useful properties of almost Hermitian $\mathcal{W}_{1}, \ldots, \mathcal{W}_{4}$ classes

Proposition 5 ([14]) We have the following characterization of Gray-Hervalla classes:

$$
\begin{aligned}
& \mathcal{W}_{1} \oplus \mathcal{W}_{2}=\left\{\xi \in T^{*} M \otimes \mathfrak{u}(n)^{\perp}(T M) \mid \xi_{J X} J Y=-\xi_{X} Y\right\} \\
& \mathcal{W}_{2} \oplus \mathcal{W}_{4}=\left\{\xi \in T^{*} M \otimes \mathfrak{u}(n)^{\perp}(T M) \mid \xi_{J X} J Y=\xi_{X} Y\right\} .
\end{aligned}
$$

Moreover,

$$
\mathcal{W}_{1} \oplus \mathcal{W}_{2} \oplus \mathcal{W}_{3}=\left\{\xi \in T^{*} M \otimes \mathfrak{u}(n)^{\perp}(T M) \mid \chi=0\right\} .
$$

By the above proposition, if $\xi \in \mathcal{W}_{1} \oplus \mathcal{W}_{2} \oplus \mathcal{W}_{3}$, then formula (10) is a point-wise formula for the $u(n)^{\perp}$-component of the scalar curvature

$$
\frac{1}{2} s_{\mathfrak{u}(n)^{\perp}}=\left|\xi^{\text {alt }}\right|^{2}-\left|\xi^{\text {sym }}\right|^{2} .
$$

The left-hand side has a nice interpretation, which is valid for all Gray-Hervella classes. Define Ricci and $*$-Ricci tensors by

$$
\operatorname{Ric}(X, Y)=\sum_{i} g\left(R\left(X, e_{i}\right) e_{i}, Y\right), \quad \operatorname{Ric}^{*}(X, Y)=g\left(R\left(X, e_{i}\right) J e_{i}, J Y\right) .
$$

These induce, taking traces, scalar curvatures

$$
s=\sum_{i} \operatorname{Ric}\left(e_{i}, e_{i}\right), \quad s^{*}=\sum_{i} \operatorname{Ric}^{*}\left(e_{i}, e_{i}\right)
$$

Then, by (16)

$$
\begin{aligned}
s_{\mathfrak{u}(n)^{\perp}} & =\sum_{i, j} g\left(R\left(e_{j}, e_{i}\right)_{\mathfrak{u}(n)^{\perp}} e_{i}, e_{j}\right) \\
& =\frac{1}{2} \sum_{i, j} g\left(R\left(e_{j}, e_{i}\right) e_{i}, e_{j}\right)+g\left(J R\left(e_{j}, e_{i}\right) J e_{i}, e_{j}\right) \\
& =\frac{1}{2}\left(s-s^{*}\right) .
\end{aligned}
$$

Now, we will discus relations between elements in the divergence formula (10) in each pure class $\mathcal{W}_{i}$ separately. We will proceed by studying quadratic invariants of the $U(n)$ representation on the space of intrinsic torsions $T^{*} M \otimes \mathfrak{u}(n)^{\perp}(T M)$ [14]:

$$
\begin{aligned}
i_{1} & =\sum_{i, j, k} \alpha\left(e_{i}, e_{j}, e_{k}\right)^{2} & i_{2} & =\sum_{i, j, k} \alpha\left(e_{i}, e_{j}, e_{k}\right) \alpha\left(e_{j}, e_{i}, e_{k}\right) \\
i_{3} & =\sum_{i, j, k} \alpha\left(e_{i}, e_{j}, e_{k}\right) \alpha\left(J e_{i}, J e_{j}, e_{k}\right) & i_{4} & =\sum_{i, j, k} \alpha\left(e_{i}, e_{i}, e_{k}\right) \alpha\left(e_{j}, e_{j}, e_{k}\right),
\end{aligned}
$$


Table 1 Quadratic invariants for Gray-Hervella classes $\mathcal{W}_{k}$

\begin{tabular}{llll}
\hline $\mathcal{W}_{1}$ & $i_{4}^{(1)}=0$, & $i_{3}^{(1)}=-i_{1}^{(1)}$, & $i_{2}^{(1)}=-i_{1}^{(1)}$ \\
$\mathcal{W}_{2}$ & $i_{4}^{(2)}=0$, & $i_{3}^{(2)}=-i_{1}^{(2)}$, & $i_{2}^{(2)}=\frac{1}{2} i_{1}^{(2)}$ \\
$\mathcal{W}_{3}$ & $i_{4}^{(3)}=0$, & $i_{3}^{(3)}=i_{1}^{(3)}$, & $i_{2}^{(3)}=0$ \\
$\mathcal{W}_{4}$ & $i_{4}^{(4)}=\frac{1}{2}(n-1) i_{1}^{(4)}$, & $i_{3}^{(4)}=i_{1}^{(4)}$, & $i_{2}^{(4)}=0$ \\
\hline
\end{tabular}

where $\alpha(X, Y, Z)=g\left(\xi_{X} Y, Z\right)$. Notice that

$$
i_{1}=|\xi|^{2}, \quad i_{2}=\left|\xi^{\mathrm{sym}}\right|^{2}-\left|\xi^{\text {alt }}\right|^{2}, \quad i_{4}=|\chi|^{2} .
$$

Thus, the divergence formula (10) may be rewritten, using these invariants and formula (22) as follows:

$$
\operatorname{div} \chi=-\frac{1}{4}\left(s-s^{*}\right)-i_{2}+i_{4}
$$

It is not hard to see by definitions of each pure class $\mathcal{W}_{i}$ and Proposition 5 that the following fact holds.

Proposition 6 Quadratic invariants characterize pure Gray-Hervella classes $\mathcal{W}_{i}$ as listed in Table 1. In a table, $i_{j}^{(k)}$ denotes an invariant $i_{j}$ considered for a class $\mathcal{W}_{k}$.

Using Proposition 6 and relation (23), we can derive some useful relations for each pure class. These relations are well known. (See the references listed in the proposition below.) Let us enlarge on this. In analogy to Gray [15], we consider the following curvature condition:

$$
\mathcal{S C}: s=s^{*} \text {. }
$$

Notice that the class $\mathcal{S C}$ contains Gray class $\mathcal{G}_{1}$, which by definition, denotes almost Hermitian structures for which the curvature tensor satisfies

$$
\mathcal{G}_{1}: \quad R(X, Y, Z, W)=R(X, Y, J Z, J W) .
$$

Proposition 7 The following relations hold.

1. (compare $[10,13])$ For a $\mathcal{W}_{1}$ structure $s-s^{*}=|\nabla J|^{2}$. In particular, there is no nearly Kähler non-Kähler structure satisfying $\mathcal{S C}$ condition.

2. (compare $[10,19])$ For a $\mathcal{W}_{2}$ structure $2\left(s-s^{*}\right)=-|\nabla J|^{2}$. In particular, there is no almost Kähler non-Kähler structure satisfying $\mathcal{S C}$ condition.

3. Any $\mathcal{W}_{3}$ structure satisfies $\mathcal{S C}$ condition.

4. (compare $[9,10,22])$ For a $\mathcal{W}_{4}$ structure with a Lee form $\theta$

$$
(n-1) \operatorname{div} \theta^{\sharp}=-\left(s-s^{*}\right)+(n-1)^{2}|\theta|^{2} .
$$

In particular, there is no locally conformally Kähler non-Kahler structure defined on a closed manifold which satisfies $\mathcal{S C}$ condition.

Proof It suffices to apply (10), Proposition 6 and use the fact that $|\xi|^{2}=\frac{1}{4}|\nabla J|^{2}$. For a $\mathcal{W}_{4}$ case, notice that

$$
\chi=\frac{n-1}{2} \theta^{\sharp}, \quad i_{4}^{(4)}=\frac{(n-1)^{2}}{4}|\theta|^{2} .
$$


Now, we show that the main integral formula (11) in an almost Hermitian case is equivalent to a Bor-Lamoneda formula [5]. Decompose $\xi$ and $\chi$ with respect to the Gray-Hervella classes as follows:

$$
\xi=\xi^{1}+\xi^{2}+\xi^{3}+\xi^{4}, \quad \chi=\chi^{1}+\chi^{2}+\chi^{3}+\chi^{4},
$$

i.e., $\chi_{k}=\sum_{i} \xi_{e_{i}}^{k} e_{i}$, and let

$$
E_{k}=\left|\chi^{k}\right|^{2}+\left|\xi^{k, \text { alt }}\right|^{2}-\left|\xi^{k, \text { sym }}\right|^{2}=-i_{2}^{(k)}+i_{4}^{(k)} .
$$

It can be shown that

$$
i_{j}=\sum_{k} i_{j}^{(k)}, \quad j=1,2,3,4
$$

Thus, by above considerations (see also Remark 1), we have

$$
E_{1}=\left|\xi^{1}\right|^{2}, \quad E_{2}=-\frac{1}{2}\left|\xi^{2}\right|^{2}, \quad E_{3}=0, \quad E_{4}=\frac{1}{2}(n-1)\left|\xi^{4}\right|^{2} .
$$

Hence,

$$
\operatorname{div} \chi=\left|\xi^{1}\right|^{2}-\frac{1}{2}\left|\xi^{2}\right|^{2}+\frac{n-1}{2}\left|\xi^{4}\right|^{2}-\frac{1}{4}\left(s-s^{*}\right),
$$

which implies the integral formula by Bor and Hernández Lamoneda [5] (assuming $M$ is closed)

$$
\int_{M}\left(2\left|\xi^{1}\right|^{2}-\left|\xi^{2}\right|^{2}+(n-1)\left|\xi^{4}\right|^{2}\right) \operatorname{vol}_{M}=\frac{1}{2} \int_{M} s-s^{*} \operatorname{vol}_{M}
$$

\subsection{Special almost Hermitian structures}

Assume $(M, g, J)$ is an almost Hermitian manifold equipped with a complex volume form $\Psi=\psi_{+}+i \psi_{-}$such that $\langle\Psi, \Psi\rangle_{\mathbb{C}}=1$, where the inner product is a natural extension of an inner product for real-valued forms. This structure defines reduction of a structure group to special unitary group $S U(n)$, hence a $S U(n)$-structure. On the level of Lie algebras, we have

$$
\mathfrak{s o}(2 n)=\mathfrak{s u}(n) \oplus\left(\mathfrak{u}(n)^{\perp} \oplus \mathbb{R}\right),
$$

since $\mathfrak{u}(n)=\mathfrak{s u}(n) \oplus \mathbb{R}$. For an element $A \in \mathfrak{u}(n)$, let

$$
A=\left(\begin{array}{cc}
A_{0} & -A_{1} \\
A_{1} & A_{0}
\end{array}\right) \in \mathfrak{s o}(2 n) .
$$

Then, $A \in \mathfrak{s u}(n)$ if and only if $A \in \mathfrak{u}(n)$ and $\operatorname{tr} A_{1}=0$. Notice that

$$
\operatorname{tr} A_{1}=\frac{1}{2} \sum_{i} g\left(A e_{i}, J e_{i}\right)=-\frac{1}{2} \operatorname{tr}(A J) .
$$

Thus, the orthogonal projection from $\mathfrak{s o}(2 n)$ to $\mathfrak{s u}(n)^{\perp}=\mathfrak{u}(n)^{\perp} \oplus \mathbb{R}$ equals

$$
A \mapsto \frac{1}{2}(A+J A J)-\frac{1}{2 n}(\operatorname{tr} A J) J .
$$


The intrinsic torsion $\xi$ equals $\xi=\xi^{U(n)}+\eta[5,8,20]$, where $\xi^{U(n)}$ is the intrinsic torsion of related $U(n)$-structure and

$$
\eta_{X} Y=-\frac{1}{2 n} \sum_{i} g\left(\xi_{X} J e_{i}, e_{i}\right) J Y .
$$

Define a one-form $\eta$ by the relation $\eta_{X} Y=\eta(J X) J Y$. This convention will appear to be useful. Denote the class in the space of all possible intrinsic torsions induced by $\eta$ by $\mathcal{W}_{5}$.

Split $s_{\mathfrak{s u}(n)^{\perp}}$ into $s_{\mathfrak{u}(n)^{\perp}}$ and $s_{\mathbb{R}}$ with respect to the decomposition $\mathfrak{s u}(n)^{\perp}=\mathfrak{u}(n)^{\perp} \oplus \mathbb{R}$.

Proposition 8 On a closed $S U(n)$-structure $(M, g, J)$ with the $\mathcal{W}_{5}$-component induced by the 1-form $\eta$, we have the following integral formula:

$$
8 \int_{M} \eta\left(\chi^{U(n)}\right) \operatorname{vol}_{M}=\int_{M} s_{\mathbb{R}}-s_{\mathfrak{s u}(n)^{\perp}}^{\text {alt }} \operatorname{vol}_{M} .
$$

In particular, if $(M, g, J)$ is of Gray-Hervella class $\mathcal{W}_{1} \oplus \mathcal{W}_{2} \oplus \mathcal{W}_{3}$ treated as $U(n)$-structure, then $\int_{M} s_{\mathbb{R}}=\int_{M} s_{\mathfrak{s u}(n) \perp}^{\text {alt }}$.

\section{Proof}

$$
\chi=\chi^{U(n)}+\sum_{i} \eta\left(J e_{i}\right) J e_{i}=\chi^{U(n)}+\eta^{\sharp}
$$

and

$$
\begin{aligned}
\sum_{i, j} g\left(\xi_{e_{i}} e_{j}, \xi_{e_{j}} e_{i}\right)= & \sum_{i, j} g\left(\xi_{e_{i}}^{U(n)} e_{j}, \xi_{e_{j}}^{U(n)} e_{i}\right)+2 \sum_{i, j} g\left(\xi_{e_{i}}^{U(n)} e_{j}, \eta\left(J e_{j}\right) J e_{i}\right) \\
& +\sum_{i, j} \eta\left(J e_{i}\right) \eta\left(J e_{j}\right) g\left(J e_{j}, J e_{i}\right) \\
= & \sum_{i, j} g\left(\xi_{e_{i}}^{U(n)} e_{j}, \xi_{e_{j}}^{U(n)} e_{i}\right)-2 \eta\left(\chi^{U(n)}\right)+\left|\eta^{\sharp}\right|^{2} .
\end{aligned}
$$

Thus,

$$
\begin{aligned}
\operatorname{div} \chi= & -\frac{1}{2} s_{\mathfrak{s u}(n)^{\perp}}+\frac{1}{2} s_{\mathfrak{s u}(n)^{\perp}}^{\mathrm{alt}}+|\chi|^{2}-\sum_{i, j} g\left(\xi_{e_{i}} e_{j}, \xi_{e_{j}} e_{i}\right) \\
= & -\frac{1}{2} s_{\mathfrak{s u}(n)^{\perp}}+\frac{1}{2} s_{\mathfrak{s u}(n)^{\perp}}^{\mathrm{alt}}+\left|\chi^{U(n)}\right|^{2}+2 \eta\left(\chi^{U(n)}\right)+\left|\eta^{\sharp}\right|^{2} \\
& -\sum_{i, j} g\left(\xi_{e_{i}}^{U(n)} e_{j}, \xi_{e_{j}}^{U(n)} e_{i}\right)+2 \eta\left(\chi^{U(n)}\right)-\left|\eta^{\sharp}\right|^{2} \\
= & \operatorname{div} \chi^{U(n)}+\frac{1}{2} s_{\mathfrak{s u}(n)^{\perp}}^{\text {alt }}-\frac{1}{2} s_{\mathfrak{s u}(n)^{\perp}}+\frac{1}{2} s_{\mathfrak{u}(n)^{\perp}}+4 \eta\left(\chi^{U(n)}\right) .
\end{aligned}
$$

Hence,

$$
\operatorname{div} \eta^{\sharp}=-\frac{1}{2} s_{\mathbb{R}}+\frac{1}{2} s_{\mathfrak{s u}(n)^{\perp}}^{\text {alt }}+4 \eta\left(\chi^{U(n)}\right) .
$$

Assuming $M$ is closed and applying the Stokes theorem, we get (26).

The values of $s_{\mathbb{R}}$ and $s_{\mathfrak{s u}(n)^{\perp}}^{\text {alt }}$ can be computed explicitly, which gives an alternative version of formula (26). Firstly, introduce two components of the intrinsic torsion, $\xi^{U(n), 12} \in$ $\mathcal{W}_{1} \oplus \mathcal{W}_{2}$ and $\xi^{U(n), 34} \in \mathcal{W}_{3} \oplus \mathcal{W}_{4}$. 
Corollary 2 On a closed $S U(n)$-structure $(M, g, J)$ with the $\mathcal{W}_{5}$-component induced by the 1-form $\eta$, we have the following integral formula

$$
\int_{M} s^{*} \operatorname{vol}_{M}=\int_{M}\left(\left|\xi^{U(n), 12}\right|^{2}-\left|\xi^{U(n), 34}\right|^{2}\right) \operatorname{vol}_{M}+4 n \int_{M} \eta\left(\chi^{U(n)}\right) \operatorname{vol}_{M} .
$$

In particular, if $\xi^{U(n)} \in \mathcal{W}_{1} \oplus \mathcal{W}_{2} \oplus \mathcal{W}_{3} \oplus \mathcal{W}_{5}$, then

$$
\int_{M} s^{*} \operatorname{vol}_{M}=\int_{M}\left|\xi^{U(n), 1}\right|^{2}+\left|\xi^{U(n), 2}\right|^{2}-\left|\xi^{U(n), 3}\right|^{2} \operatorname{vol}_{M}
$$

Proof We have

$$
\begin{aligned}
s_{\mathbb{R}} & =\sum_{i, j} g\left(R\left(e_{i}, e_{j}\right)_{\mathbb{R}} e_{j}, e_{i}\right) \\
& =-\frac{1}{2 n} \sum_{i, j} \operatorname{tr}\left(R\left(e_{i}, e_{j}\right) J\right) g\left(J e_{j}, e_{i}\right) \\
& =-\frac{1}{2 n} \sum_{i, j} g\left(R\left(J e_{j}, e_{j}\right) J e_{i}, e_{i}\right) \\
& =\frac{1}{2 n} \sum_{i, j}\left(g\left(R\left(J e_{i}, J e_{j}\right) e_{j}, e_{i}\right)+g\left(R\left(e_{j}, J e_{i}\right) J e_{j}, e_{i}\right)\right) \\
& =\frac{1}{n} s^{*} .
\end{aligned}
$$

To compute $s_{\mathfrak{s u}(n)^{\perp}}^{\text {alt }}$, it is convenient to determine the component $\left[\mathfrak{s u}(n)^{\perp}, \mathfrak{s u}(n)^{\perp}\right]_{\mathfrak{s u}(n)^{\perp}}$. For $A=A_{0}+\lambda J$ and $B=B_{0}+\mu J$, where $A_{0}, B_{0} \in \mathfrak{u}(n)^{\perp}$, by the relation $\left[\mathfrak{u}(n)^{\perp}, \mathfrak{u}(n)^{\perp}\right] \subset$ $\mathfrak{u}(n)$, we have

$$
\begin{aligned}
{[A, B]_{\mathfrak{s u}(n)^{\perp}} } & =\left[A_{0}, B_{0}\right]_{\mathbb{R}}+\lambda\left[J, B_{0}\right]+\mu\left[A_{0}, J\right] \\
& =-\frac{1}{n} \operatorname{tr}\left(A_{0} B_{0} J\right) J+2\left(\mu A_{0}-\lambda B_{0}\right) J .
\end{aligned}
$$

Hence,

$$
\begin{aligned}
s_{\mathfrak{s u}(n)^{\perp}}^{\mathrm{alt}} & =\sum_{i, j}\left(-\frac{1}{n} \operatorname{tr}\left(\xi_{e_{i}} \xi_{e_{j}} J\right) g\left(J e_{j}, e_{i}\right)+2 g\left(\eta\left(J e_{j}\right) \xi_{e_{i}} J e_{j}-\eta\left(J e_{i}\right) \xi_{e_{j}} J e_{j}, e_{i}\right)\right) \\
& =-\frac{1}{n} \sum_{i, j} g\left(\xi_{J e_{j}} \xi_{e_{j}} J e_{i}, e_{i}\right)+2 \sum_{i, j}\left(\eta\left(J e_{j}\right) g\left(\xi_{e_{i}} J e_{j}, e_{i}\right)-\eta\left(J e_{i}\right) g\left(\xi_{e_{j}} J e_{j}, e_{i}\right)\right) \\
& =\frac{1}{n} \sum_{i, j} g\left(\xi_{e_{j}} J e_{i}, \xi_{J e_{j}} e_{i}\right)-4 \eta\left(\chi^{U(n)}\right) \\
& =-\frac{1}{n} \sum_{i, j} g\left(\xi_{e_{j}} e_{i}, \xi_{J e_{j}} J e_{i}\right)-4 \eta\left(\chi^{U(n)}\right) .
\end{aligned}
$$

By Proposition 5, we get

$$
\sum_{i, j} g\left(\xi_{e_{j}} e_{i}, \xi_{J e_{j}} J e_{i}\right)=-\left|\xi^{U(n), 12}\right|^{2}+\left|\xi^{U(n), 34}\right|^{2} .
$$

Now, it suffices to apply (26). 
Remark 2 The above integral formula, however formulated in a different way, can be found in [5]. Let us be more precise. In [5], authors state some consequences of their formula for almost Hermitian structures with vanishing first Chern class $c_{1}(M)$. Let us derive the first Chern class in our setting. It is known [13] that the first Chern form $\gamma$ is given by

$$
8 \pi \gamma=-\varphi+2 \psi,
$$

where

$$
\varphi(X, Y)=\operatorname{tr}\left(\left(\nabla_{X} J\right)\left(\nabla_{Y} J\right)\right), \quad \psi(X, Y)=\operatorname{tr}(R(X, Y) \circ J) .
$$

It is not hard to see that

$$
\varphi(X, Y)=4 \sum_{i} g\left(\xi_{X} J e_{i}, \xi_{Y} e_{i}\right), \quad \psi(X, Y)=-2 \operatorname{Ric}^{*}(X, J Y) .
$$

Thus, using the same arguments as before Corollary 2, we get

$$
2 \pi \operatorname{tr}^{*} \gamma=\left|\xi^{U(n), 34}\right|^{2}-\left|\xi^{U(n), 12}\right|^{2}+s^{*},
$$

where $\operatorname{tr}^{*} \gamma=\sum_{i} \gamma\left(e_{i}, J e_{i}\right)$. Notice that vanishing of the first Chern class, i.e., $\gamma=d \alpha$ for some 1-form, is equivalent to the fact that $\int_{M} \operatorname{tr}^{*} \gamma=0$. Thus, by Corollary $2, c_{1}(M)=0$ if and only if $\int_{M} \eta\left(\chi^{U(n)}\right)=0$. Finally, note that by (2), we have

$$
\int_{M} \eta\left(\chi^{U(n)}\right) \operatorname{vol}_{M}=-\int_{M} \operatorname{div}^{G} \eta^{\sharp} \operatorname{vol}_{M} .
$$

Corollary 3 Consider an $S U(n)$-structure $(M, g, J)$ which is of type $\mathcal{W}_{1} \oplus \mathcal{W}_{5}$. Then,

$$
\int_{M} s \operatorname{vol}_{M}=5 \int_{M} s^{*} \operatorname{vol}_{M}
$$

Proof By Corollary 2, we have

$$
\int_{M} s^{*}=\int_{M}\left|\xi^{U(n)}\right|^{2}
$$

It suffices to notice that by (22) and (21), we have $\frac{1}{4}\left(s-s^{*}\right)=\left|\xi^{U(n)}\right|^{2}$.

\subsection{Almost contact metric structures}

Let $(M, g)$ be a $(2 n+1)$-dimensional manifold together with a 1-form $\eta$ (and its dual unit vector field $\zeta$ ) and $\varphi \in \operatorname{End}(T M)$ such that

$$
\varphi^{2} X=-X+\eta(X) \zeta, \quad g(\varphi X, \varphi Y)=g(X, Y)-\eta(X) \eta(Y) .
$$

Notice that $\varphi$ defines almost complex structure which is $g$-orthogonal on the distribution ker $\eta$. Thus, we get $U(n) \times 1$-structure. On the level of Lie algebras, we have

$$
\mathfrak{s o}(2 n+1)=\mathfrak{u}(n) \oplus \mathfrak{u}(n)^{\perp},
$$

where $\mathfrak{u}(n)^{\perp}$ is isomorphic to the space of block matrices of the form

$$
\left(\begin{array}{cc}
B & a \\
-a^{\top} & 0
\end{array}\right), \quad B \in \mathfrak{u}(n)^{\perp} \subset \mathfrak{s o}(2 n), \quad a \in \mathbb{R}^{2 n} .
$$


Since here $\zeta=e_{2 n+1}, \varphi$ is a natural complex structure on $\mathbb{R}^{2 n}$ and zero on $\zeta$, it is easy to see that the orthogonal projection from $\mathfrak{s o}(2 n+1)$ onto $\mathfrak{u}(n)^{\perp}$ equals

$$
A \mapsto \frac{1}{2}\left(A+\varphi A \varphi+\zeta^{\top} A \otimes \zeta+\zeta^{\top} \otimes A \zeta\right) .
$$

Rewriting this formula with the use of the one-form $\eta\left(\equiv \zeta^{\top}\right)$, the intrinsic torsion satisfies the following relation:

$$
\xi_{X} Y=\varphi\left(\xi_{X} \varphi Y\right)+\eta\left(\xi_{X} Y\right) \zeta+\eta(Y) \xi_{X} \zeta .
$$

This, moreover, implies the formula for the intrinsic torsion [12]

$$
\xi_{X} Y=\frac{1}{2}\left(\nabla_{X} \varphi\right) \varphi Y+\frac{1}{2}\left(\nabla_{X} \eta\right) Y \cdot \zeta-\eta(Y) \nabla_{X} \zeta
$$

By (28), it follows that

$$
\varphi\left(\nabla_{X} \varphi\right) Y=-\left(\nabla_{X} \varphi\right) \varphi Y+\left(\nabla_{X} \eta\right) Y \cdot \zeta+\eta(Y) \nabla_{X} \zeta .
$$

Thus, we may write the intrinsic torsion in an alternative way [12]

$$
\xi_{X} Y=-\frac{1}{2} \varphi\left(\nabla_{X} \varphi\right) Y+\left(\nabla_{X} \eta\right) Y \cdot \zeta-\frac{1}{2} \eta(Y) \nabla_{X} \eta
$$

Hence, the characteristic vector field in this case equals

$$
\chi=-\frac{1}{2} \varphi(\operatorname{div} \varphi)+(\operatorname{div} \zeta) \zeta-\frac{1}{2} \nabla_{\zeta} \zeta .
$$

The condition $\xi \in T^{*} M \otimes \mathfrak{u}(n)^{\perp}(T M)$ is equivalent to relation (31). Decomposing the space $T^{*} M \otimes \mathfrak{u}(n)^{\perp}(T M)$ into irreducible $U(n) \times 1$-modules, we get 12 classes $\mathcal{C}_{1}, \ldots, \mathcal{C}_{12}$ [7]. First four are isomorphic to Gray-Hervella classes $\mathcal{W}_{1}, \ldots, \mathcal{W}_{4}$.

Remark 3 Note that in [7] types of almost contact metric structures, i.e., irreducible modules of $T^{*} M \otimes \mathfrak{u}(n)^{\perp}(T M)$, were classified with respect to $\alpha(X, Y, Z)=\left(\nabla_{X} \Phi\right)(Y, Z)$, where $\Phi(X, Y)=g(X, \varphi Y)$. It is well known that this is equivalent to considering the intrinsic torsion as a map $\beta(X, Y, Z)=g\left(\xi_{X} Y, Z\right)$. The correspondence follows from the fact that $\nabla_{X} \Phi=\xi_{X} \Phi$, since $\nabla^{U(n) \times 1} \Phi=0$. This implies a direct relation

$$
\alpha(X, Y, Z)=\beta(X, Y, \varphi Z)-\beta(X, Z, \varphi Y) .
$$

Note that we should be careful with studying irreducible modules $\mathcal{C}_{1}, \ldots, \mathcal{C}_{12}$, since the correspondence $\alpha \leftrightarrow \beta$ interchanges some of the modules, which is underlined in Table 2 .

Let us describe these spaces in more detail. Put

$$
\mathcal{D}_{1}=\mathcal{C}_{1} \oplus \ldots \oplus \mathcal{C}_{4}, \quad \mathcal{D}_{2}=\mathcal{C}_{5} \oplus \ldots \oplus \mathcal{C}_{11}, \quad \mathcal{D}_{3}=\mathcal{C}_{12}
$$

Each of the above spaces is characterized as follows [7]:

1. Class $\mathcal{D}_{1}: \xi_{\zeta} Y=\xi_{X} \zeta=0$. Applying formluas (32) and (33), we obtain $\nabla \zeta=0$ and hence $\chi=-\frac{1}{2} \varphi(\operatorname{div} \varphi)$, as expected, since in this case, being not very precise, $\xi$ is the intrinsic torsion on the almost Hermitian structure ker $\eta$.

Table 2 Module correspondence via $\alpha \leftrightarrow \beta$

\begin{tabular}{lllllllllllll}
\hline$\alpha$ & $\mathcal{C}_{1}$ & $\mathcal{C}_{2}$ & $\mathcal{C}_{3}$ & $\mathcal{C}_{4}$ & $\mathcal{C}_{5}$ & $\mathcal{C}_{6}$ & $\mathcal{C}_{7}$ & $\mathcal{C}_{8}$ & $\mathcal{C}_{9}$ & $\mathcal{C}_{10}$ & $\mathcal{C}_{11}$ & $\mathcal{C}_{12}$ \\
$\beta$ & $\mathcal{C}_{1}$ & $\mathcal{C}_{2}$ & $\mathcal{C}_{3}$ & $\mathcal{C}_{4}$ & $\mathcal{C}_{6}$ & $\mathcal{C}_{5}$ & $\mathcal{C}_{8}$ & $\mathcal{C}_{7}$ & $\mathcal{C}_{9}$ & $\mathcal{C}_{10}$ & $\mathcal{C}_{11}$ & $\mathcal{C}_{12}$ \\
\hline
\end{tabular}


2. Class $\mathcal{D}_{2}: \xi_{X} Y=\eta(X) \xi_{\zeta} Y+\eta(Y) \xi_{X} \zeta+\eta\left(\xi_{X} Y\right) \zeta$. Taking $X=Y=\zeta$, we get $\xi_{\zeta} \zeta=-\eta\left(\xi_{\zeta} \zeta\right) \zeta$ and $\eta$ to both sides we get $\xi_{\zeta} \zeta=0$. Taking $X=Y=e_{i}$, we obtain

$$
\operatorname{pr}_{\mathrm{ker} \eta} \chi=0 \text {. }
$$

Hence, $\chi \in \operatorname{span} \zeta$, so by (34), $\chi=(\operatorname{div} \zeta) \zeta$. Moreover, it is easy to see that for $X, Y \in \operatorname{ker} \eta$

$$
\xi_{X} Y \in \operatorname{span} \zeta, \quad \xi_{\zeta} Y \in \operatorname{ker} \eta, \quad \xi_{X} \zeta \in \operatorname{ker} \eta, \quad \xi_{\zeta} \zeta=0 .
$$

Thus, by formulas (32) and (33) for the intrinsic torsion, we get ( $X, Y \in \operatorname{ker} \eta)$

$$
\begin{aligned}
\xi_{X} Y & =\left(\nabla_{X} \eta\right) Y \cdot \zeta, \\
\xi_{\zeta} Y & =\frac{1}{2}\left(\nabla_{\zeta} \varphi\right) \varphi Y, \\
\xi_{X} \zeta & =-\nabla_{X} \zeta .
\end{aligned}
$$

3. Class $\mathcal{D}_{3}: \xi_{X} Y=\eta(X) \eta(Y) \xi_{\zeta} \zeta+\eta(X) \eta\left(\xi_{\zeta} Y\right) \zeta$. Therefore, for $X, Y \in \operatorname{ker} \eta$

$$
\xi_{X} Y=0, \quad \xi_{X} \zeta=0, \quad \xi_{\zeta} Y \in \operatorname{span} \zeta, \quad \xi_{\zeta} \zeta \in \operatorname{ker} \eta .
$$

Hence, $\chi=\xi_{\zeta} \zeta=-\nabla_{\zeta} \zeta$ and $|\chi|^{2}+\left|\xi^{\text {alt }}\right|^{2}-\left|\chi^{\text {sym }}\right|^{2}=0$.

Analogously as in an almost Hermitian case, let us relate divergence and integral formulas (10), (11) to quadratic invariants of almost contact metric structure. In this case, the space of quadratic invariants is generated by 18 invariants [7]. For our purposes, we need only some of them:

$$
\begin{aligned}
i_{1} & =\sum_{i, j, k} \alpha\left(e_{i}, e_{j}, e_{k}\right)^{2}, & i_{2} & =\sum_{i, j, k} \alpha\left(e_{i}, e_{j}, e_{k}\right) \alpha\left(e_{j}, e_{i}, e_{k}\right)^{2}, \\
i_{4} & =\sum_{i, j, k} \alpha\left(e_{i}, e_{i}, e_{k}\right) \alpha\left(e_{j}, e_{j}, e_{k}\right), & i_{5} & =\sum_{j, k} \alpha\left(\zeta, e_{j}, e_{k}\right)^{2}, \\
i_{6} & =\sum_{i, j, k} \alpha\left(e_{i}, \zeta, e_{k}\right)^{2}, & i_{7} & =\sum_{j, k} \alpha\left(\zeta, e_{j}, e_{k}\right) \alpha\left(e_{j}, \zeta, e_{k}\right), \\
i_{8} & =\sum_{i, j} \alpha\left(e_{i}, e_{j}, \zeta\right) \alpha\left(e_{j}, e_{i}, \zeta\right), & i_{10} & =\sum_{i, j} \alpha\left(e_{i}, e_{i}, \zeta\right) \alpha\left(e_{j}, e_{j}, \zeta\right), \\
i_{12} & =\sum_{i, j} \alpha\left(e_{i}, e_{j}, \zeta\right) \alpha\left(\varphi\left(e_{j}\right), \varphi\left(e_{i}\right), \zeta\right), & i_{14} & =\sum_{i, j} \alpha\left(e_{i}, \varphi\left(e_{i}\right), \zeta\right) \alpha\left(e_{j}, \varphi\left(e_{j}\right), \zeta\right), \\
i_{16} & =\sum_{k} \alpha\left(\zeta, \zeta, e_{k}\right)^{2}, & i_{17} & =\sum_{i, k} \alpha\left(e_{i}, e_{i}, e_{k}\right) \alpha\left(\zeta, \zeta, e_{k}\right),
\end{aligned}
$$

where $\alpha(X, Y, Z)=g\left(\xi_{X} Y, Z\right)$ and $i, j=1, \ldots, 2 n$. Notice that

$$
\begin{aligned}
|\xi|^{2} & =i_{1}+i_{5}+2 i_{6}+2 i_{16}, \\
|\chi|^{2} & =i_{4}+i_{10}+i_{16}, \\
\left|\xi^{\mathrm{sym}}\right|^{2}-\left|\xi^{\mathrm{alt}}\right|^{2} & =i_{2}+i_{7}+i_{8}+i_{16} .
\end{aligned}
$$

Let us now compute scalar curvature 'components'. Define the $*$-scalar curvature as follows:

$$
s^{*}=\operatorname{tr} \operatorname{Ric}^{*}=\sum_{i, j} g\left(R\left(e_{i}, e_{j}\right) \varphi e_{j}, \varphi e_{i}\right) .
$$


Lemma 1 The following relations hold:

$$
\begin{aligned}
s_{\mathfrak{u}(n)^{\perp}}^{\text {alt }} & =\frac{1}{2}\left(i_{8}-i_{10}+i_{12}+i_{14}\right)+2\left(i_{7}-i_{17}\right) \text { and } \\
s_{\mathfrak{u}(n)^{\perp}} & =\frac{1}{2}\left(s-s^{*}\right)+\operatorname{Ric}(\zeta, \zeta) .
\end{aligned}
$$

Proof Denote by $(B, a)$ an element in $\mathfrak{u}(n)^{\perp}$ of form (29). Note that $a=(B, a) e_{2 n+1} \in$ $\mathbb{R}^{2 n} \subset \mathbb{R}^{2 n+1}$. Then,

$$
[(B, a),(\tilde{B}, \tilde{a})]=(-a \wedge \tilde{a}, B \tilde{a}-\tilde{B} a),
$$

where $a \wedge \tilde{a}$ is an endomorphism of $\mathbb{R}^{2 n}$ given by $(a \wedge \tilde{a}) v=\langle\tilde{a}, v\rangle a-\langle a, v\rangle \tilde{a}$. Thus

$$
[(B, a),(\tilde{B}, \tilde{a})]_{\mathfrak{u}(n)^{\perp}}=\left(-\frac{1}{2}\left(a \wedge \tilde{a}+\varphi_{0}(a \wedge \tilde{a}) \varphi_{0}, B \tilde{a}-\tilde{B} a\right),\right.
$$

where $\varphi_{0}$ is a restriction of $\varphi$ to $\mathbb{R}^{2 n}$, thus defining an almost complex structure.

By these considerations, we are ready to compute $s_{\mathfrak{u}(n)^{\perp}}^{\text {alt }}$. We have (here $i, j=1, \ldots, 2 n$ )

$$
\begin{aligned}
s_{\mathfrak{u}(n)^{\perp}}^{\mathrm{alt}}= & -\frac{1}{2} \sum_{i, j} g\left(\left(\xi_{e_{i}} z \wedge \xi_{e_{j}} z+\varphi\left(\xi_{e_{i}} z \wedge \xi_{e_{j}} z\right) \varphi\right) e_{j}, e_{i}\right)+2 \sum_{i} g\left(\xi_{e_{i}} \xi_{\zeta} \zeta-\xi_{\zeta} \xi_{e_{i}} \zeta, e_{i}\right) \\
= & -\frac{1}{2} \sum_{i, j}\left(g\left(\xi_{e_{i}} e_{i}, \zeta\right) g\left(\xi_{e_{j}} e_{i}, \zeta\right)-g\left(\xi_{e_{i}} e_{j}, \zeta\right) g\left(\xi_{e_{j}} e_{i}, \zeta\right)\right. \\
& \left.-g\left(\xi_{e_{i}} \varphi\left(e_{i}\right), \zeta\right) g\left(\xi_{e_{j}} \varphi\left(e_{j}\right), \zeta\right)+g\left(\xi_{e_{i}} \varphi\left(e_{j}\right), \zeta\right) g\left(\xi_{e_{j}} \varphi\left(e_{i}\right), \zeta\right)\right) \\
& +2 \sum_{i}\left(-g\left(\xi_{\zeta} \zeta, \xi_{e_{i}} e_{i}\right)+g\left(\xi_{e_{i}} \zeta, \xi_{\zeta} e_{i}\right)\right) \\
= & \frac{1}{2}\left(-i_{10}+i_{8}+i_{14}+i_{12}\right)+2\left(-i_{17}+i_{7}\right) .
\end{aligned}
$$

For $s_{\mathfrak{u}(n)^{\perp}}$ by (30), we have

$$
\begin{aligned}
s_{\mathfrak{u}(n)^{\perp}} & =\sum_{i, j} g\left(R\left(e_{i}, e_{j}\right)_{\mathfrak{u}(n)^{\perp}} e_{j}, e_{i}\right) \\
& =\frac{1}{2}\left(s-s^{*}\right)+\frac{1}{2} \sum_{i, j}\left(\eta\left(R\left(e_{i}, e_{j}\right) e_{j}\right) \eta\left(e_{i}\right)+\eta\left(e_{j}\right) g\left(R\left(e_{i}, e_{j}\right) \zeta, e_{i}\right)\right. \\
& =\frac{1}{2}\left(s-s^{*}\right)+\sum_{i}\left(g\left(R\left(\zeta, e_{j}\right) e_{j}, \zeta\right)\right. \\
& =\frac{1}{2}\left(s-s^{*}\right)+\operatorname{Ric}(\zeta, \zeta) .
\end{aligned}
$$

By Lemma 1 and above considerations, we may rewrite formula (10) as

$$
\operatorname{div} \chi=-i_{2}+i_{4}-\frac{3}{4} i_{8}+\frac{3}{4} i_{10}+\frac{1}{4} i_{12}-\frac{1}{4} i_{14}-i_{17}-\frac{1}{4}\left(s-s^{*}\right)-\frac{1}{2} \operatorname{Ric}(\zeta, \zeta)
$$

Moreover, by a classification of each module $\mathcal{C}_{i}$ by quadratic invariants [7, Table I], we have the following observation. 
Proposition 9 We have:

1. the characteristic vector field $\chi$ vanishes if and only if the intrinsic torsion belongs to $\mathcal{C}_{1} \oplus \mathcal{C}_{2} \oplus \mathcal{C}_{3} \oplus \mathcal{C}_{6} \oplus \mathcal{C}_{7} \oplus \mathcal{C}_{8} \oplus \mathcal{C}_{9} \oplus \mathcal{C}_{10} \oplus \mathcal{C}_{11}$

2. $\left|\xi^{\mathrm{sym}}\right|^{2}-\left|\xi^{\text {alt }}\right|^{2}=0$ if and only if the intrinsic torsion belongs to $\mathcal{C}_{3} \oplus \mathcal{C}_{4} \oplus \mathcal{C}_{11}$,

3. $s_{\mathfrak{u}(n)^{\perp}}^{\text {alt }}=0$ if and only if the intrinsic torsion belongs to $\mathcal{C}_{1} \oplus \mathcal{C}_{2} \oplus \mathcal{C}_{3} \oplus \mathcal{C}_{4} \oplus \mathcal{C}_{7} \oplus \mathcal{C}_{9} \oplus$ $\mathcal{C}_{10} \oplus \mathcal{C}_{11} \oplus \mathcal{C}_{12}$

We are ready to interpret, at least in some cases, formula (36) in a geometric way. Denote by $B^{\eta}$ and $T^{\eta}$ the (symmetric) second fundamental form and integrability tensor of ker $\eta$, respectively (see the section on almost product structures).

Proposition 10 Let $(M, g, \varphi, \eta, \zeta)$ be an almost contact metric structure with the intrinsic torsion $\xi$.

1. If $\xi \in \mathcal{D}_{2}$, then

$$
\operatorname{div}((\operatorname{div} \zeta) \zeta)=(\operatorname{div} \zeta)^{2}+\left|T^{\eta}\right|^{2}-\left|B^{\eta}\right|^{2}+\frac{1}{2} s_{\mathfrak{u}(n)^{\perp}}^{\text {alt }}-\frac{1}{4}\left(s-s^{*}\right)-\frac{1}{2} \operatorname{Ric}(\zeta, \zeta),
$$

or, equivalently,

$$
\operatorname{div}\left(\nabla_{\zeta} \zeta\right)=\frac{1}{2} s_{\mathfrak{u}(n)^{\perp}}^{\text {alt }}+\frac{1}{2} \operatorname{Ric}(\zeta, \zeta)-\frac{1}{4}\left(s-s^{*}\right) .
$$

If additionally, $M$ is closed, then the following integral formula holds

$$
\int_{M} \operatorname{Ric}(\zeta, \zeta) \operatorname{vol}_{M}=\frac{1}{2} \int_{M} s-s^{*}-2 s_{\mathfrak{u}(n)^{\perp}}^{\mathrm{alt}} \operatorname{vol}_{M}=\int_{M}(\operatorname{div} \zeta)^{2}+\left|T^{\eta}\right|^{2}-\left|B^{\eta}\right|^{2} \operatorname{vol}_{M} .
$$

2. If $\xi \in \mathcal{C}_{6} \oplus \ldots \oplus \mathcal{C}_{11}$, then

$$
\left|T^{\eta}\right|^{2}-\left|B^{\eta}\right|^{2}=-\frac{1}{2} s_{\mathfrak{u}(n)^{\perp}}^{\text {alt }}+\frac{1}{4}\left(s-s^{*}\right)+\frac{1}{2} \operatorname{Ric}(\zeta, \zeta)
$$

3. If $\xi \in \mathcal{C}_{11}$, then $s-s^{*}=-2 \operatorname{Ric}(\zeta, \zeta)$ and $\int_{M} s-s^{*} \operatorname{vol}_{M}=\int_{M} \operatorname{Ric}(\zeta, \zeta) \operatorname{vol}_{M}=0$.

4. If $\xi \in \mathcal{C}_{12}$, then

$$
\operatorname{div}\left(\nabla_{\zeta} \zeta\right)=\frac{1}{4}\left(s-s^{*}\right)+\frac{1}{2} \operatorname{Ric}(\zeta, \zeta)
$$

If, additionally, $M$ is closed, then

$$
\int_{M} s-s^{*} \operatorname{vol}_{M}=-2 \int_{M} \operatorname{Ric}(\zeta, \zeta) \text { vol. }
$$

Proof (1) Assume $\xi \in \mathcal{D}_{2}$. Then, for $X, Y \in \operatorname{ker} \eta$, we have

$$
\xi_{X}^{\text {alt }} Y=-T^{\eta}(X, Y), \quad \xi_{X}^{\text {sym }} Y=-B^{\eta}(X, Y) .
$$

Moreover, by classification of $\mathcal{D}_{2}$ by quadratic invariants [7], we see that

$$
\left|\xi^{\text {alt }}\right|^{2}-\left|\xi^{\text {sym }}\right|^{2}=-i_{8}=\left|T^{\eta}\right|^{2}-\left|B^{\eta}\right|^{2} .
$$

Since $\chi=(\operatorname{div} \zeta) \zeta$ using Proposition 4 , the divergence formula (10) takes the first form. The second one and the integral formula follow again by Proposition 4.

(2) In this case, by Proposition 9, the characteristic vector field vanishes, i.e., div $\zeta=0$. Thus, it suffices to apply the first part. 
(3) This is an immediate consequence of (1), (2) and the fact that $\left|T^{\eta}\right|^{2}-\left|B^{\eta}\right|^{2}$ and $s_{\mathfrak{u}(n)^{\perp}}^{\text {alt }}$ vanish (by Proposition 9).

(4) By discussion concerning $\mathcal{D}_{3}$ class, we have $|\chi|^{2}+\left|\xi^{\text {alt }}\right|^{2}-\left|\xi^{\text {sym }}\right|^{2}=0$ and $\chi=-\nabla_{\zeta} \zeta$. Moreover, by Proposition 9, $s_{\mathfrak{u}(n)^{\perp}}^{\text {alt }}=0$. Hence, the divergence formula (10) and the integral formula (11) simplify to the desired ones. Notice that we could use the formula (36) and the fact that for $C_{12}$ class the only nonzero invariant is $i_{16}$ [7, Table I].

At the end, consider the following Janssen-Vanhecke $\mathcal{C}(\alpha)$ condition [17]:

$$
\begin{aligned}
R(X, Y, Z, W)= & R(X, Y, \varphi Z, \varphi W)+\alpha(-g(X, Z) g(Y, W)+g(X, W) g(Y, Z) \\
& +g(X, \varphi Z) g(Y, \varphi W)-g(X, \varphi W) g(Y, \varphi Z)),
\end{aligned}
$$

where $\alpha$ is a smooth function. It implies that

$$
s-s^{*}=4 n^{2} \alpha \text { and } \operatorname{Ric}(\zeta, \zeta)=2 n \alpha .
$$

Corollary 4 If the intrinsic torsion of an almost contact metric structure belongs to $\mathcal{D}_{2}$ class and satisfies Janssen-Vanhecke $C(\alpha)$ condition, then

$$
\alpha=\frac{1}{n(n-1)}\left(\frac{1}{2} s_{\mathfrak{u}(n)^{\perp}}^{\text {alt }}-\operatorname{div}\left(\nabla_{\zeta} \zeta\right)\right) .
$$

Proof Follows immediately by Proposition 10(1).

Remark 4 In an analogous way as for $U(n)$-structures, it can be shown, with a little bit more effort, that the integral formula (11) in this case is equivalent to the integral formula obtained in [12].

\section{Examples}

In this section, we apply obtained results to certain almost Hermitian and almost contact metric structures. First examples are simple illustration of obtained results for almost contact metric structures. We deal with this structure only since it has not been investigated from this point of view elsewhere. Almost Hermitian case is, due to well-known facts contained in Proposition 7, well understood from this perspective. In the end, we focus on more involving examples concerning homogeneous spaces, where we treat both cases-almost Hermitian and almost contact metric structures.

Example 1 Let $(M, g, \varphi, \eta, \zeta)$ be an almost contact metric structure which is Sasaki, i.e., $\varphi$ satisfies the relation

$$
\left(\nabla_{X} \varphi\right) Y=g(X, Y) \xi-\eta(Y) X, \quad \nabla_{X} \zeta=-\varphi(X) .
$$

Thus, by (32), the intrinsic torsion equals

$$
\xi_{X} Y=g(X, \varphi(Y)) \zeta
$$

or, in matrix notation

$$
\xi_{X}=\left(\begin{array}{cc}
0 & \varphi(X) \\
-\varphi(X)^{\top} & 0
\end{array}\right) \in \mathfrak{u}(n)^{\perp}(T M) \text { for } X \in \operatorname{ker} \eta
$$


and $\xi_{\zeta}=0$. Hence, we arrive in the $\mathcal{C}_{5}$ class. From the proof of Lemma 1 or, directly, by Proposition 9, we see that $s_{\mathfrak{u}(n)^{\perp}}^{\text {alt }}$ does not vanish. In fact, by [7, Table 1], we have

$$
i_{6}=-i_{8}=-i_{12}=\frac{1}{2 n} i_{14}
$$

Thus, by the definition of $i_{6}$ and Lemma 1

$$
s_{\mathfrak{u}(n)^{\perp}}^{\text {alt }}=(n-1) i_{6}=2 n(n-1) .
$$

It can be shown [17] that the curvature tensor of a Sasakian structure satisfies $C(1)$ condition. Thus, by (37), $s-s^{*}=4 n^{2}$ and $\operatorname{Ric}(\zeta, \zeta)=2 n$. Since $\nabla_{\zeta} \zeta=-\varphi(\zeta)=0$, it follows that the left-hand side of the second divergence formula in Proposition 10(1) vanishes, whereas the right-hand side equals

$$
\frac{1}{2} s_{\mathfrak{u}(n)^{\perp}}^{\text {alt }}-\frac{1}{4}\left(s-s^{*}\right)+\frac{1}{2} \operatorname{Ric}(\zeta, \zeta)=n(n-1)-n^{2}+n=0 .
$$

Example 2 Let $(M, g, \varphi, \eta, \zeta)$ be an almost contact metric structure which is Kenmotsu, i.e., $\varphi$ satisfies the following condition

$$
\left(\nabla_{X} \varphi\right) Y=g(\varphi X, Y) \zeta-\eta(Y) \varphi(X) .
$$

Hence, the intrinsic torsion, by the formula (32), equals

$$
\xi_{X} Y=g(X, Y) \zeta, \quad \xi_{X} \zeta=-X, \quad \xi_{\zeta} Y=0, \quad \xi_{\zeta} \zeta=0,
$$

where $X, Y \in \operatorname{ker} \eta$. In matrix notation, for $X \in \operatorname{ker} \eta$,

$$
\xi_{X}=\left(\begin{array}{cc}
0 & X \\
-X^{\top} & 0
\end{array}\right) \in \mathfrak{u}(n)^{\perp}(T M) .
$$

Hence, arguing as in the example above, $s_{\mathfrak{u}(n)^{\perp}}^{\text {alt }}$ does not vanish. Since, in this case,

$$
i_{6}=i_{8}=i_{12}=\frac{1}{2 n} i_{10}
$$

by Lemma 1 ,

$$
s_{\mathfrak{u}(n)^{\perp}}^{\text {alt }}=(1-n) i_{6}=2 n(1-n) .
$$

It can be shown that Kenmotsu structure satisfies $C(-1)$ condition [17]. By (37), $s-s^{*}=$ $-4 n^{2}$ and $\operatorname{Ric}(\zeta, \zeta)=-2 n$. Therefore,

$$
\frac{1}{2} s_{\mathfrak{u}(n)^{\perp}}^{\text {alt }}-\frac{1}{4}\left(s-s^{*}\right)+\frac{1}{2} \operatorname{Ric}(\zeta, \zeta)=n(1-n)+n^{2}-n=0 .
$$

Since for a Kenmotsu manifold $\nabla_{X} \zeta=X-\eta(X) \zeta$, it follows that

$$
\operatorname{div} \zeta=2 n \text { and } \nabla_{\zeta} \zeta=0
$$

It follows that the second divergence formula in Proposition 10 is justified.

Let us now justify condition (2) in Proposition 10. It is known that the distribution ker $\eta$ is integrable and umbilical [18]. Hence, $T^{\eta}=0$ and $B^{\eta}=-\frac{1}{2 n} g \otimes(\operatorname{div} \zeta) \zeta$, which implies

$$
\left|T^{\eta}\right|^{2}-\left|B^{\eta}\right|^{2}=-2 n
$$

and

$$
\frac{1}{4}\left(s-s^{*}\right)+\frac{1}{2} \operatorname{Ric}(\zeta, \zeta)-\frac{1}{2} s_{\mathfrak{u}(n)^{\perp}}^{\mathrm{alt}}=-n^{2}-n-n(1-n)=-2 n
$$


Example 3 Consider a generalized Heisenberg group $H(1, n)$ [12]. This is a Lie group consisting of square $n+2$ by $n+2$ matrices of the form

$$
g=\left(\begin{array}{ccc}
I_{n} & A^{t} & B^{t} \\
0 & 1 & c \\
0 & 0 & 1
\end{array}\right)
$$

where $A=\left(a_{1}, \ldots, a_{n}\right)$ and $B=\left(b_{1}, \ldots, b_{n}\right)$ are elements of $\mathbb{R}^{n}$ and $c \in \mathbb{R}$. Then, $H(1, n)$ is nilpotent of dimension $2 n+1$. $H(1, n)$ has a global coordinate system $\left(x^{i}, x^{n+1}, z\right)$, $i=1,2, \ldots, n$ given by

$$
x^{i}(g)=a_{i}, \quad x^{n+i}(g)=b_{i}, \quad z(g)=c .
$$

We choose a Riemannian metric such that the left-invariant basis

$$
X_{i}=\frac{\partial}{\partial x^{i}}, \quad Y_{i}=\frac{\partial}{\partial x^{n+i}}, \quad Z=\frac{\partial}{\partial z}+\sum_{i} x^{i} \frac{\partial}{\partial x^{n+i}}
$$

is an orthonormal one. Nonzero components of the Levi-Civita connection are given by [12]

$$
\begin{aligned}
& \nabla_{X_{i}} X_{n+i}=\nabla_{X_{n+i}} X_{i}=-\frac{1}{2} Z, \\
& \nabla_{X_{i}} Z=-\nabla_{Z} X_{i}=\frac{1}{2} X_{n+1}, \\
& \nabla_{X_{n+i}} Z=\nabla_{Z} X_{n+i}=\frac{1}{2} X_{i} .
\end{aligned}
$$

Thus, the integrability tensor $T^{\eta}$ vanishes and nonzero components of the second fundamental form are equal

$$
B^{\eta}\left(X_{i}, X_{n+i}\right)=B^{\eta}\left(X_{n+i}, X_{i}\right)=-\frac{1}{2} Z
$$

Hence, $\left|B^{\eta}\right|^{2}=\frac{n}{2}$. Moreover, by the formula for the curvature tensor [12]

$$
\begin{array}{ll}
R\left(X_{i}, X_{j}, X_{n+i}, X_{n+j}\right)=\frac{1}{4} i \neq j, & R\left(X_{i}, X_{n+j}, X_{j}, X_{n+i}\right)=-\frac{1}{4}, \\
R\left(X_{i}, Z, X_{i}, Z\right)=\frac{3}{4}, & R\left(X_{n+i}, Z, X_{n+i}, Z\right)=-\frac{1}{4}
\end{array}
$$

with remaining components vanishing, we see that

$$
s=-\frac{n}{2}, \quad \operatorname{Ric}(Z, Z)=-\frac{n}{2} .
$$

Consider on $H(1, n)$ an almost contact structure induced by the Reeb field $\zeta=Z$ and a dual one form $\eta=d z$ with a compatible endomorphism $\varphi$ such that $\varphi\left(X_{i}\right)=X_{n+i}$, $\varphi\left(X_{n+1}\right)=-X_{i}$. Then, we get a structure in a $\mathcal{C}_{9}$ class [12]. We have

$$
s^{*}=\frac{n}{2} \text {. }
$$

It is easy to see that $\operatorname{div} Z=0$; hence, the characteristic vector field vanishes (as noticed in Proposition 9(1)). We are ready to compute both sides of the divergence formula in Proposition 10(2). The left-hand side, clearly, equals $-\frac{n}{2}$. Since by Proposition 9(3), $s_{\mathfrak{u}(n)^{\perp}}^{\text {alt }}=0$, the right-hand side is equal to

$$
\frac{1}{4}\left(s-s^{*}\right)+\frac{1}{2} \operatorname{Ric}(\zeta, \zeta)=-\frac{n}{4}-\frac{n}{4}=-\frac{n}{2} .
$$




\subsection{Examples on reductive homogeneous spaces}

We will show that for a certain choice of $G$-structures on reductive homogeneous spaces induced from one-parameter deformations of invariant Riemannian metrics, the characteristic vector field $\chi$ vanishes; hence, the divergence formula becomes point-wise formula. We justify this stating appropriate examples. We closely follow [3, p. 140] and [1].

Let $K$ be a connected, compact Lie group and $H$ its closed, connected Lie subgroup. The quotient $K / H$ is a homogeneous space denoted by $M$. Assume additionally that on the level of Lie algebras $\mathfrak{k}=\mathfrak{h} \oplus \mathfrak{m}$, where $\mathfrak{m}$ is the orthogonal complement with respect to some $\operatorname{ad}(H)$-invariant bilinear form $\mathbf{B}$ on $\mathfrak{k}$. Deform $\mathbf{B}$ in the following way: Assume $\mathfrak{m}=\mathfrak{m}_{0} \oplus \mathfrak{m}_{1}$, where

$$
\begin{aligned}
& {\left[\mathfrak{h}, \mathfrak{m}_{0}\right]=\mathfrak{m}_{0}, \quad\left[\mathfrak{m}_{0}, \mathfrak{m}_{0}\right] \subset \mathfrak{h} \oplus \mathfrak{m}_{1},} \\
& {\left[\mathfrak{h}, \mathfrak{m}_{1}\right] \subset \mathfrak{m}_{1}, \quad\left[\mathfrak{m}_{1}, \mathfrak{m}_{1}\right] \subset \mathfrak{h}, \quad\left[\mathfrak{m}_{0}, \mathfrak{m}_{1}\right] \subset \mathfrak{m}_{0} .}
\end{aligned}
$$

For any $t>0$, we put

$$
\mathbf{B}_{t}=\left.\mathbf{B}\right|_{\mathfrak{m}_{0} \times \mathfrak{m}_{0}}+\left.2 t \mathbf{B}\right|_{\mathfrak{m}_{1} \times \mathfrak{m}_{1}} .
$$

Form $\mathbf{B}_{t}$ defines an invariant Riemannian metric $g_{t}$ on $M$. We will often write $g$ instead of $g_{t}$, if there is no confusion. The Levi-Civita connection of $g$ may be described as a linear map $\Lambda: \mathfrak{m} \rightarrow \mathfrak{s o}(\mathfrak{m})$ defined as follows: [3]

$$
\begin{aligned}
\Lambda(X) Y & =\frac{1}{2}[X, Y]_{\mathfrak{m}_{1}}, \\
\Lambda(X) B & =t[X, B], \\
\Lambda(A) Y & =(1-t)[A, Y], \\
\Lambda(A) B & =0,
\end{aligned}
$$

where $X, Y \in \mathfrak{m}_{0}, A, B \in \mathfrak{m}_{1}$.

Now, we consider a $G$-structure on $M$. Thus, we have a decomposition $\mathfrak{s o}(\mathfrak{m})=\mathfrak{g} \oplus \mathfrak{g}^{\perp}$, where we take orthogonal complement with respect to the Killing form on $\mathfrak{s o}(\mathfrak{m})$. Then, $\Lambda$ splits as $\Lambda=\Lambda_{\mathfrak{g}}+\Lambda_{\mathfrak{g}^{\perp}} \cdot \Lambda_{\mathfrak{g}}$ defines a $G$-connection $\nabla^{G}$, whereas $\Lambda_{\mathfrak{g}^{\perp}}$ corresponds to the intrinsic torsion $\xi$.

In the following two examples, we introduce a $G$-structure via the same procedure. Denote by $x_{0}$ the coset $e H$, and let ad $: H \rightarrow \mathrm{SO}(\mathfrak{m})$ be the isotropy representation. Let $\varphi: \mathfrak{m} \rightarrow \mathfrak{m}$ be a linear map, which intertwines the isotropy representation. Since all tensor bundles on $M$ are associated with the bundle $G \mapsto M$ with respect to the isotropy representation, it follows that $\varphi$ induces $(1,1)$-tensor field, in our case, almost Hermitian or almost contact metric structure.

Example 4 We follow [3, p. 142]. Consider a complex flag manifold $F_{1,2}$ consisting of pairs $(l, V)$, where $l$ is one-dimensional complex subspace and $V$ is a complex two-dimensional subspace containing $l$ in $\mathbb{C}^{3}$. $U(3)$ acts transitively with a isotropy subgroup $H=U(1) \times$ $U(1) \times U(1)$. Thus, $F_{1,2}$ is a homogeneous space. On the level of Lie algebras, $\mathfrak{u}(n)=\mathfrak{h} \oplus \mathfrak{m}$, where $\mathfrak{h}$ consists of diagonal matrices, whereas $\mathfrak{m}$ is a subspace of the form

$$
\mathfrak{m}=\left\{\left(\begin{array}{ccc}
0 & a & b \\
-\bar{a} & 0 & c \\
-\bar{b} & -\bar{c}
\end{array}\right), \quad a, b, c \in \mathbb{C}\right\} .
$$


$\mathfrak{m}$ splits into two subspaces $\mathfrak{m}_{0}$ and $\mathfrak{m}_{1}$ given, respectively, by relations $c=0$ and $a=b=0$. Denote by $\mathbf{B}$ the Killing form on $\left.\mathfrak{u}(3), \mathbf{B}(X, Y)=\frac{1}{2} \operatorname{Re}(\operatorname{tr} X Y)\right)$. The inner product on $\mathfrak{m}$ given by

$$
-\left.\mathbf{B}\right|_{\mathfrak{m}_{0} \times \mathfrak{m}_{0}}+\left.2 t(-\mathbf{B})\right|_{\mathfrak{m}_{1} \times \mathfrak{m}_{1}}
$$

defines a one-parameter family of Riemannian metrics on $F_{1,2}$. The following basis is orthonormal with respect to the given inner product on $\mathfrak{m}$ :

$$
E_{1}=e_{12}, \quad E_{2}=s_{12}, \quad E_{3}=e_{13}, \quad E_{4}=s_{13}, \quad E_{5}=\frac{1}{\sqrt{2 t}} e_{23}, \quad E_{6}=\frac{1}{\sqrt{2 t}} s_{23},
$$

where $e_{j k}$ is a skew-symmetric matrix with the $(j, k)$ entry equal to 1 and $s_{j k}$ is a symmetric matrix with the $(j, k)$-entry equal to $i$ (and remaining elements except for $(k, j)$-entry equal to zero). We see that $\mathfrak{m}_{0}$ is spanned by $E_{1}, E_{2}, E_{3}, E_{4}$, whereas $\mathfrak{m}_{1}$ by $E_{5}, E_{6}$. It can be verified that relations (39) hold.

Let us define an almost Hermitian structure on $F_{1,2}$. The isotropy representation Ad : $H \rightarrow \mathrm{SO}(\mathfrak{m})=\mathrm{SO}(6)$ equals

$$
\operatorname{Ad}(t, r, s)=\left(\begin{array}{ccc}
R_{t-s} & 0 & 0 \\
0 & R_{t-r} & 0 \\
0 & 0 & R_{s-r}
\end{array}\right)
$$

where $(t, r, s)$ denotes an element $\operatorname{diag}\left(e^{i t}, e^{i s}, e^{i r}\right) \in H$ and $R_{\theta}$ is a rotation in $\mathbb{R}^{2}$ through an angle $\theta$. In order to define an almost Hermitian structure, it suffices to define isotropy invariant $(1,1)$-tensor $J_{0}$ in $\mathfrak{m}$ with $J_{0}^{2}=-1$. Let

$$
J_{0}\left(E_{1}\right)=-E_{2}, \quad J_{0}\left(E_{3}\right)=E_{4}, \quad J_{0}\left(E_{5}\right)=-E_{6} .
$$

The Levi-Civita connection of this almost Hermitian structure can be described by a map $\Lambda: \mathfrak{m} \rightarrow \mathfrak{s o}(\mathfrak{m})$,

$$
\begin{aligned}
\Lambda\left(E_{1}\right) & =\frac{\sqrt{t}}{\sqrt{2}}\left(e_{35}+e_{46}\right), & & \Lambda\left(E_{2}\right)=\frac{\sqrt{t}}{\sqrt{2}}\left(e_{45}-e_{36}\right), \\
\Lambda\left(E_{3}\right) & =\frac{\sqrt{t}}{\sqrt{2}}\left(e_{26}-e_{15}\right), & \Lambda\left(E_{4}\right) & =-\frac{\sqrt{t}}{\sqrt{2}}\left(e_{16}+e_{25}\right), \\
\Lambda\left(E_{5}\right) & =\frac{1-t}{\sqrt{2 t}}\left(e_{13}+e_{24}\right), & \Lambda\left(E_{6}\right) & =\frac{1-t}{\sqrt{2 t}}\left(e_{14}-e_{23}\right) .
\end{aligned}
$$

The curvature tensor $R$ is given by

$$
R(X, Y)=[\Lambda(X), \Lambda(Y)]-\Lambda\left([X, Y]_{\mathfrak{m}}\right)-\operatorname{Ad}\left([X, Y]_{\mathfrak{h}}\right),
$$

where Ad $: \mathfrak{h} \rightarrow \mathfrak{s o}(\mathfrak{m})$ denotes the differential of the isotropy representation,

$$
\operatorname{Ad}\left(H_{1}\right)=-e_{12}-e_{34}, \quad \operatorname{Ad}\left(H_{2}\right)=e_{12}-e_{56}, \quad \operatorname{Ad}\left(H_{3}\right)=e_{34}+e_{56} .
$$

Here, $H_{k}$ denotes the matrix $\frac{1}{2} s_{k k}$.

Now we are ready to compute $s_{\mathfrak{u}(3)^{\perp}}$. Deriving relations for the commutators in $\mathfrak{m}$ and its components in $\mathfrak{h}$ and then the curvature tensor $R$ and projections of elements $e_{i j}$ to $\mathfrak{u}(3)^{\perp}$, we get

$$
s_{\mathfrak{u}(3)^{\perp}}=8(2-t) .
$$


We could obtain the above relation by applying formula (22). It is easy to see that $s=$ $2\left(-13+3 t-\frac{2}{t}\right)$ and $s^{*}=2\left(3-5 t-\frac{2}{t}\right)$.

Let us turn to computations of the intrinsic torsion and its components. We easily see that $\Lambda: \mathfrak{m} \rightarrow \mathfrak{u}(3)^{\perp}$. Hence, the minimal connection $\nabla^{U(3)}$ is induced by a zero map. Moreover,

$$
\left.\sum_{i, j}\left\langle\Lambda\left(E_{i}\right) E_{i}, \Lambda\left(E_{j}\right) E_{i}\right)\right\rangle=4(t-2)
$$

corresponds to $\left|\xi^{\mathrm{sym}}\right|^{2}-\left|\xi^{\text {alt }}\right|^{2}$; hence, the main divergence formula, which reduces to $\frac{1}{2} s_{\mathfrak{u}(3)^{\perp}}=\left|\xi^{\text {alt }}\right|^{2}-\left|\xi^{\text {sym }}\right|^{2}=4(2-t)$, is justified.

Let us look at the Gray-Hervella classes induced by $t$ for each choice of $t>0$. Since $\chi=0$, the considered almost Hermitian structure is of type $\mathcal{W}_{1} \oplus \mathcal{W}_{2} \oplus \mathcal{W}_{3}$. Simple calculations show that $\Lambda$, hence $\xi$, satisfies $\xi_{J X} J Y=-\xi_{X} Y$. Thus, by Proposition 5, the considered structures are of type $\mathcal{W}_{1} \oplus \mathcal{W}_{2}$. Moreover, it is nearly Kähler, i.e., in $\mathcal{W}_{1}$, if and only if $t=\frac{1}{2}$. By above considerations, we see that for $t<2, s_{\mathfrak{u}(3) \perp}>0$ and for $t>2$, $s_{\mathfrak{u}(3) \perp}<0$.

Example 5 We follow very closely the approach by Agricola [1]. Consider the fivedimensional Stiefel manifolds $V_{4,2}=S O(4) / S O(2)$. We embed $S(2)$ as a lower diagonal block. We have the splitting $\mathfrak{s o}(4)=\mathfrak{s o}(2) \oplus \mathfrak{m}$ with respect to the Killing form $\mathbf{B}$, where

$$
\mathfrak{m}=\left\{\left(\begin{array}{cc}
A & X \\
-X^{\top} & 0
\end{array}\right) \mid A=\left(\begin{array}{cc}
0 & -a \\
a & 0
\end{array}\right), \quad X \in \mathcal{M}_{2 \times 2}(\mathbb{R})\right\} .
$$

There is a one-parameter family $g_{t}$ of Riemannian metrics on $V_{4,2}$ constructed by Jensen [16], which are obtained from the invariant dot product on $\mathfrak{m}$

$$
\langle(a, X),(b, Y)\rangle=\mathbf{B}(X, Y)+2 t a b,
$$

where $(a, X),(b, Y)$ denote the elements in $\mathfrak{m}$ and $\mathbf{B}(X, Y)=\frac{1}{2} \operatorname{tr}\left(X^{\top} Y\right)$. Denoting the canonical basis in $\mathfrak{s o}(4)$ by $\left(e_{i j}\right)$, i.e., $e_{i j}$ is a skew-symmetric matrix with the $(i, j)$-entry equal to -1 (be aware of the difference with the sign convention compared to the previous example), we have an orthonormal basis

$$
E_{1}=e_{13}, \quad E_{2}=e_{14}, \quad E_{3}=e_{23}, \quad E_{4}=e_{24}, \quad E_{5}=\frac{1}{\sqrt{2 t}} e_{12}
$$

in $\mathfrak{m}$. The Levi-Civita connection of this homogeneous space is described by a map $\Lambda: \mathfrak{m} \rightarrow$ $\mathfrak{s o}(\mathfrak{m})=\mathfrak{s o}(5)$ of the form $[1,16]$

$$
\begin{aligned}
& \Lambda\left(E_{1}\right)=\sqrt{\frac{t}{2}} e_{35}, \quad \Lambda\left(E_{2}\right)=\sqrt{\frac{t}{2}} e_{45}, \quad \Lambda\left(e_{3}\right)=-\sqrt{\frac{t}{2}} e_{15}, \\
& \Lambda\left(E_{4}\right)=-\sqrt{\frac{t}{2}} e_{25}, \quad \Lambda\left(E_{5}\right)=\frac{1-t}{\sqrt{2 t}}\left(e_{13}+e_{24}\right) .
\end{aligned}
$$

The curvature tensor is then given by

$$
R(X, Y)=[\Lambda(X), \Lambda(Y)]-\Lambda\left([X, Y]_{\mathfrak{m}}\right)-\operatorname{Ad}\left([X, Y]_{\mathfrak{h}}\right),
$$

where Ad is the differential of the isotropy representation Ad $: S O(2) \rightarrow S O(\mathfrak{m})$,

$$
\operatorname{Ad}(g)=\left(\begin{array}{lll}
g & 0 & 0 \\
0 & g & 0 \\
0 & 0 & 0
\end{array}\right), \quad g \in S O(2) .
$$


Now we introduce an almost contact structure on $V_{4,2}$ by defining an isotropy invariant $\operatorname{map} \varphi: \mathfrak{m} \rightarrow \mathfrak{m}$, which is, in terms of the basis $\left(E_{i}\right)$, given by a matrix

$$
\varphi=\left(\begin{array}{ccccc}
0 & 0 & 1 & 0 & 0 \\
0 & 0 & 0 & 1 & 0 \\
-1 & 0 & 0 & 0 & 0 \\
0 & -1 & 0 & 0 & 0 \\
0 & 0 & 0 & 0 & 0
\end{array}\right)
$$

One can check that $\varphi$, in deed, defines an almost contact structure with the Reeb field $\zeta$ induced by $E_{5}$ and compatible with the metric $g_{t}$. Notice that the fundamental form $F(X, Y)=$ $g_{t}(X, \varphi(Y))$ induced by $\varphi$ if $F=e_{13}+e_{24}$, which is proportional to $d E_{5}$ making the structure just Sasaki structure [1].

To derive the formula for $s_{\mathfrak{u}(2)^{\perp}}$, we need to compute the $\mathfrak{u}(2)^{\perp}$-component of $R$, i.e., project $R\left(E_{i}, E_{j}\right)$ to the second factor with respect to the decomposition $\mathfrak{s o}(\mathfrak{m})=\mathfrak{u}(2) \oplus$ $\mathfrak{u}(2)^{\perp}$. It is easy to see that $e_{i 5} \in \mathfrak{u}(2)^{\perp}$ and $e_{13}, e_{24} \in \mathfrak{u}(2)$. Moreover, $e_{13}$ and $e_{24}$ project to $v=\frac{1}{2}\left(e_{13}-e_{24}\right)$ and $-v$, respectively. Finally, $e_{12}$ projects to $w=\frac{1}{2}\left(e_{12}-e_{34}\right)$, whereas $e_{34}$ projects to $-w$. Thus,

$$
s_{\mathfrak{u}(2) \perp}=6 t .
$$

We could compute $s_{\mathfrak{u}(2)^{\perp}}$ using Lemma 1 and noticing that

$$
s=2(4-t), \quad s^{*}=2(4-5 t), \quad \operatorname{Ric}(\zeta, \zeta)=2 t .
$$

Let us describe the intrinsic torsion. We see that $\Lambda_{\mathfrak{u}(3)^{\perp}}\left(E_{i}\right)=\Lambda\left(E_{i}\right)$ for $i=1,2,3,4$, and $\Lambda_{\mathfrak{u}(3)^{\perp}}\left(E_{5}\right)=0$. Hence, the characteristic vector field $\chi$, which corresponds to $\sum_{i} \Lambda_{\mathfrak{u}(2)^{\perp}}\left(E_{i}\right) E_{i}$, vanishes and, it is not hard to see that $\left|\xi^{\text {alt }}\right|^{2}-\left|\xi^{\text {sym }}\right|^{2}$, which corresponds to $-\sum_{i, j}\left\langle\Lambda_{\mathfrak{u}(2) \perp}\left(E_{i}\right) E_{j}, \Lambda_{\mathfrak{u}(2)^{\perp}}\left(E_{j}\right) E_{i}\right\rangle$, equals $2 t$. Finally, directly from the definition, it is not hard to see that $s_{\mathfrak{u}(2)^{\perp}}^{\text {alt }}=2 t$. Thus,

$$
\frac{1}{2} s_{\mathfrak{u}(2)^{\perp}}^{\text {alt }}-\frac{1}{2} s_{\mathfrak{u}(2)^{\perp}}+\left|\xi^{\text {alt }}\right|^{2}-\left|\xi^{\text {sym }}\right|^{2}=t-3 t+2 t=0 .
$$

Notice, finally, that by the classification of all possible intrinsic torsion modules [7], since $i_{2}=\left|\xi^{\text {sym }}\right|^{2}-\left|\xi^{\text {alt }}\right|=-t<0$, we arrive in a pure $\mathcal{C}_{1}$ class.

Acknowledgements I wish to thank Ilka Agricola for indication of references [4] and [1] and helpful conversations. The author is partially supported by the National Science Center, Poland-Grant Miniatura 2017/01/X/ST1/01724.

Open Access This article is distributed under the terms of the Creative Commons Attribution 4.0 International License (http://creativecommons.org/licenses/by/4.0/), which permits unrestricted use, distribution, and reproduction in any medium, provided you give appropriate credit to the original author(s) and the source, provide a link to the Creative Commons license, and indicate if changes were made.

\section{References}

1. Agricola, I.: Connections on naturally reductive spaces, their Dirac operator and homogeneous models in string theory. Comm. Math. Phys. 232(3), 535-563 (2003)

2. Agricola, I.: The Srni lectures on non-integrable geometries with torsion. Arch. Math. (Brno) 42(suppl.), 5-84 (2006)

3. Baum, H., Friedrich, T., Grunewald, R., Kath, I.: Twistors and Killing Spinors on Riemannian Manifolds, Teubner Texts in Mathematics. Stuttgart (1991) 
4. Berger, M., Gauduchon, P., Mazet, E.: Le Spectre d'une Variété Riemannienne. Lecture Notes in Mathematics. Springer, Berlin (1977)

5. Bor, G., Hernández Lamoneda, L.: Bochner formulae for orthogonal G-structures on compact manifolds. Differential Geom. Appl. 15(3), 265-286 (2001)

6. Bor, G., Hernández Lamoneda, L.: A Bochner formula for almost-quaternionic-Hermitian structures. Differential Geom. Appl. 21(1), 79-92 (2004)

7. Chinea, D., González-Dávila, J.C.: A classification of almost contact metric manifolds. Ann. Mat. Pura Appl. (4) 156, 15-36 (1990)

8. Chiossi, S., Salamon, S.: The intrinsic torsion of SU(3) and G2 structures. In: Differential Geometry, Valencia, 2001, pp. 115-133. World Science Publications, River Edge, NJ (2002)

9. Falcitelli, M., Farinola, A.: Curvature properties of the locally conformal Kähler manifolds. Rend. Math. 11, 495-521 (1991)

10. Falcitelli, M., Farinola, A.: Curvature properties of almost Hermitian manifolds. Riv. Math. Univ. Parma (5) 3, 301-320 (1994)

11. Fernández, M., Gray, A.: Riemannian manifolds with structure group G2. Ann. Mat. Pura Appl. (4) 132(1982), 19-45 (1983)

12. González-Dávila, J.C., Martin Cabrera, F.: Harmonic almost contact structures via the intrinsic torsion. Israel J. Math. 181, 145-187 (2011)

13. Gray, A.: The structure of nearly Kähler manifolds. Math. Ann. 223(3), 233-248 (1976)

14. Gray, A., Hervella, L.: The sixteen classes of almost Hermitian manifolds and their linear invariants. Ann. Mat. Pura Appl. (4) 123, 35-58 (1980)

15. Gray, A.: Curvature identities for Hermitian and almost Hermitian manifolds. Tohoku Math. J. 28, 601612 (1976)

16. Jensen, G.: Imbeddings of Stiefel manifolds into Grassmannians. Duke Math. J. 42(3), 397-407 (1975)

17. Janssens, D., Vanhecke, L.: Almost contact structures and curvature tensors. Kodai Math. J. 4, 1-27 (1981)

18. Kim, T., Pak, H.: Canonical foliations of certain classes of almost contact metric structures. Acta Math. Sin. (Engl. Ser.) 21(4), 841-846 (2005)

19. Koto, S.: Some theorems on almost kählerian spaces. J. Math. Soc. Japan 12, 422-433 (1960)

20. Martin Cabrera, F.: Special almost Hermitian geometry. J. Geom. Phys. 55(4), 450-470 (2005)

21. Swann, A.: Aspects symplectiques de la géométrie quaternionique. C. R. Acad. Sci. Paris Sér. I Math. 308(7), 225-228 (1989)

22. Vaisman, I.: Some curvature properties of Locally conformal Kähler manifolds. Trans. Amer. Math. Soc. 259(2), 439-447 (1980)

23. Walczak, P.: An integral formula for a Riemannian manifold with two orthogonal complementary distributions. Colloq. Math. 58(2), 243-252 (1990)

Publisher's Note Springer Nature remains neutral with regard to jurisdictional claims in published maps and institutional affiliations. 